\title{
NON-COOPERATIVE FISHER-KPP SYSTEMS: ASYMPTOTIC BEHAVIOR OF TRAVELING WAVES
}

\author{
LÉO GIRARDIN
}

\begin{abstract}
This paper is concerned with non-cooperative parabolic reaction-diffusion systems which share structural similarities with the scalar Fisher-KPP equation. In a previous paper, we established that these systems admit traveling wave solutions whose profiles connect the null state to a compact subset of the positive cone. The main object of the present paper is the investigation of a more precise description of these profiles. Non-cooperative KPP systems can model various phenomena where the following three mechanisms occur: local diffusion in space, linear cooperation and superlinear competition.
\end{abstract}

\section{INTRODUCTION}

This paper is a sequel to a previous paper by the same author [16] where the so-called KPP systems were investigated. The prototypical and, arguably, most famous KPP system is the Lotka-Volterra mutation-competition-diffusion system:

$$
\frac{\partial \mathbf{u}}{\partial t}-\operatorname{diag}(\mathbf{d}) \Delta_{x} \mathbf{u}=\operatorname{diag}(\mathbf{r}) \mathbf{u}+\mathbf{M u}-\operatorname{diag}(\mathbf{u}) \mathbf{C u}
$$

where $\mathbf{u}$ is a nonnegative vector containing phenotypical densities, $\mathbf{d}$ and $\mathbf{r}$ are positive vectors containing respectively diffusion rates and growth rates, $\mathbf{M}$ is an essentially nonnegative irreducible matrix with null Perron-Frobenius eigenvalue containing mutation rates (typically a discrete Neumann Laplacian) and $\mathbf{C}$ is a positive matrix containing competition rates. Although the Lotka-Volterra competition-diffusion system (without mutations) is a very classical research subject, mutations can dramatically influence some of its properties and their overall effect is still poorly understood.

More generally, KPP systems as defined in [16] are non-cooperative (or nonmonotone, i.e. they do not satisfy a comparison principle; see Protter-Weinberger 30. Chapter 3, Section 8]) and have started to attract attention relatively recently. Their study requires innovative ideas and the literature is limited; a detailed bibliography can be found in [16].

By adapting proofs and methods well-known in the context of the scalar KPP equation,

$$
\frac{\partial u}{\partial t}-d \Delta_{x} u=r u-c u^{2},
$$

2000 Mathematics Subject Classification. 34D23, 35K40, 35K57, 92D25.

Key words and phrases. KPP nonlinearities, reaction-diffusion system, steady states, structured population, traveling waves.

This work has been carried out in the framework of the NONLOCAL project (ANR-14-CE250013) funded by the French National Research Agency (ANR).

Laboratoire Jacques-Louis Lions, CNRS UMR 7598, Université Pierre et Marie Curie, 4 place Jussieu, 75005 Paris, France. 
first studied by Fisher [11] and Kolmogorov, Petrovsky and Piskunov [23], various properties of these systems were established in [16]. In particular, a KPP system equipped with a reaction term sufficiently analogous to $u-u^{2}$ admits traveling wave solutions with a half-line of possible speeds and a positive minimal speed $c^{\star}$. These traveling waves are defined in a very general way: it is merely required that they describe the invasion of $\mathbf{0}$ by a positive population density. A very natural subsequent question is that of the evolution of the distribution $\mathbf{u}$ during the invasion. Which components lead the invasion? Which components settle once the invasion is over?

Having in mind that the waves traveling at speed $c^{\star}$ should attract front-like and compactly supported initial data (although this statement has yet to be proven, since [16] only established the equality between $c^{\star}$ and the spreading speed associated with such initial data, and it is expected to be a very difficult problem), a more general question is then: given a class of initial data, what is the long-time distribution of the solution?

In the rest of the introduction, we present more precisely the problem and state our main results. Sections 2,3 and 4 are dedicated to the proofs of these results. Finally, open questions, interesting remarks and numerical experiments are discussed in Section 5.

1.1. The non-cooperative KPP system. From now on, an integer $N \geq 2$ is fixed.

A positive vector $\mathbf{d} \in \mathrm{K}^{++}$, a square matrix $\mathbf{L} \in \mathrm{M}$ and a vector field $\mathbf{c} \in$ $\mathscr{C}^{1}\left(\mathbb{R}^{N}, \mathbb{R}^{N}\right)$ are fixed. We denote for the sake of brevity $\mathbf{D}=\operatorname{diag}(\mathbf{d})$.

We consider the following semilinear parabolic system:

$$
\partial_{t} \mathbf{u}-\mathbf{D} \partial_{x x} \mathbf{u}=\mathbf{L u}-\mathbf{c}[\mathbf{u}] \circ \mathbf{u}, \quad\left(E_{K P P}\right)
$$

\begin{tabular}{|c|c|}
\hline Notation & Definition \\
\hline$[n]$ & {$[1, n] \cap \mathbb{N}$} \\
\hline$\left(\mathbf{e}_{n, i}\right)_{i \in[n]}$ & canonical basis of $\mathbb{R}^{n}$ \\
\hline$|\bullet|_{n}$ & Euclidean norm of $\mathbb{R}^{n}$ \\
\hline $\mathrm{B}_{n}(\mathbf{v}, r), \mathrm{S}_{n}(\mathbf{v}, r)$ & open ball and sphere of center $\mathbf{v} \in \mathbb{R}^{n}$ and radius $r>0$ \\
\hline$\geq_{n},>_{n}, \gg_{n}$ & $v_{i} \geq \hat{v}_{i}$ for all $i \in[n], \mathbf{v} \geq_{n} \hat{\mathbf{v}}$ and $\mathbf{v} \neq \hat{\mathbf{v}}, v_{i}>\hat{v}_{i}$ for all $i \in[n]$ \\
\hline nonnegative, nonneg. nonzero, positive $\mathbf{v} \in \mathbb{R}^{n}$ & $\mathbf{v} \geq_{n} \mathbf{0}, \mathbf{v}>_{n} \mathbf{0}, \mathbf{v} \gg_{n} \mathbf{0}$ \\
\hline $\mathrm{K}_{n}, \mathrm{~K}_{n}^{+}, \mathrm{K}_{n}^{++}$ & sets of all nonnegative, nonneg. nonzero, positive vectors \\
\hline $\mathrm{S}_{n}^{+}(\mathbf{0}, 1), \mathrm{S}_{n}^{++}(\mathbf{0}, 1)$ & $\mathrm{K}_{n}^{+} \cap \mathrm{S}_{n}(\mathbf{0}, 1), \mathrm{K}_{n}^{++} \cap \mathrm{S}_{n}(\mathbf{0}, 1)$ \\
\hline $\mathrm{M}_{n, n^{\prime}}, \mathrm{M}_{n}$ & sets of all real matrices of dimension $n \times n^{\prime}, n \times n$ \\
\hline $\mathbf{I}_{n}, \mathbf{1}_{n, n^{\prime}}$ & identity matrix, matrix whose every entry is equal to 1 \\
\hline $\operatorname{diag}(\mathbf{v})$ & diagonal matrix whose $i$-th diagonal entry is $v_{i}$ \\
\hline essentially nonnegative matrix & matrix $\mathbf{A}$ such that $\mathbf{A}-\min _{i \in[n]}\left(a_{i, i}\right) \mathbf{I}_{n}$ is nonnegative \\
\hline $\mathbf{A} \circ \mathbf{B}$ & Hadamard (entry-by-entry) product $\left(a_{i, j} b_{i, j}\right)_{(i, j) \in[n] \times\left[n^{\prime}\right]}$ \\
\hline $\mathbf{f}[\hat{\mathbf{f}}]$ & composition of the functions $\mathbf{f}$ and $\hat{\mathbf{f}}$ \\
\hline
\end{tabular}

TABle 1. General notations ( the subscripts depending only on 1 or $N$ are omitted when the context is unambiguous) 
with $\mathbf{u}:(t, x) \in \mathbb{R}^{2} \mapsto \mathbf{u}(t, x) \in \mathbb{R}^{N}$ as unknown. In order to ease the notations, we only consider one-dimensional spaces, however all forthcoming results could be applied directly to traveling plane waves in multidimensional spaces (these solutions being in fact one-dimensional).

When restricted to solutions $\mathbf{u}: \mathbb{R} \rightarrow \mathbb{R}^{N}$ which are constant in space, $\left(E_{K P P}\right)$ reduces to

$$
\mathbf{u}^{\prime}=\mathbf{L u}-\mathbf{c}[\mathbf{u}] \circ \mathbf{u} . \quad\left(E_{K P P}^{0}\right)
$$

When restricted to solutions $\mathbf{u}: \mathbb{R} \rightarrow \mathbb{R}^{N}$ which are constant in time, $\left(E_{K P P}\right)$ reduces to

$$
-\mathbf{D u}^{\prime \prime}=\mathbf{L u}-\mathbf{c}[\mathbf{u}] \circ \mathbf{u} . \quad\left(S_{K P P}\right)
$$

When restricted to traveling solutions of the form $\mathbf{u}:(t, x) \mapsto \mathbf{p}(x-c t)$ with $c \in \mathbb{R},\left(E_{K P P}\right)$ reduces to

$$
-\mathbf{D} \mathbf{p}^{\prime \prime}-c \mathbf{p}^{\prime}=\mathbf{L} \mathbf{p}-\mathbf{c}[\mathbf{p}] \circ \mathbf{p} . \quad(T W[c])
$$

1.1.1. Basic KPP assumptions. The basic assumptions introduced in [16] are the following ones.

$\left(H_{1}\right) \mathbf{L}$ is essentially nonnegative and irreducible.

$\left(H_{2}\right) \mathbf{c}(\mathrm{K}) \subset \mathrm{K}$.

$\left(H_{3}\right) \mathbf{c}(\mathbf{0})=\mathbf{0}$.

$\left(H_{4}\right)$ There exists

$$
(\underline{\alpha}, \delta, \underline{\mathbf{c}}) \in[1,+\infty)^{2} \times \mathrm{K}^{++}
$$

such that

$$
\sum_{j=1}^{N} l_{i, j} n_{j} \geq 0 \Longrightarrow \alpha^{\delta} \underline{c}_{i} \leq c_{i}(\alpha \mathbf{n})
$$

for all

$$
(\mathbf{n}, \alpha, i) \in \mathrm{S}^{+}(\mathbf{0}, 1) \times[\underline{\alpha},+\infty) \times[N] .
$$

The assumption $\left(H_{4}\right)$ loosely means that $\mathbf{c}$ grows at least linearly at infinity. The precise condition means, however, that in the set $\left\{\mathbf{v} \in \mathrm{K} \mid(\mathbf{L v})_{i}<0\right\}$ (which is nonempty if and only if $l_{i, i}<0$ and contains in such a case the open half-line $\left.\operatorname{span}\left(\mathbf{e}_{i}\right) \cap \mathrm{K}^{+}\right)$, the growth of $c_{i}$ is not important. Anyway, $\left(H_{4}\right)$ includes the Lotka-Volterra form of competition (linear and positive c) as well as more general forms (see for instance Gilpin-Ayala [15]).

Recall from the Perron-Frobenius theorem that if $\mathbf{L}$ is nonnegative and irreducible, its spectral radius $\rho(\mathbf{L})$ is also its dominant eigenvalue, called the Perron-Frobenius eigenvalue $\lambda_{P F}(\mathbf{L})$, and is the unique eigenvalue associated with a positive eigenvector. Recall also that if $\mathbf{L}$ is essentially nonnegative and irreducible, the Perron-Frobenius theorem can still be applied. In such a case, the unique eigenvalue of $\mathbf{L}$ associated with a positive eigenvector is $\lambda_{P F}(\mathbf{L})=$ $\rho\left(\mathbf{L}-\min _{i \in[N]}\left(l_{i, i}\right) \mathbf{I}_{N}\right)+\min _{i \in[N]}\left(l_{i, i}\right)$. Any eigenvector associated with $\lambda_{P F}(\mathbf{L})$ is referred to as a Perron-Frobenius eigenvector and the unit one is denoted $\mathbf{n}_{P F}(\mathbf{L})$.

In view of [16. Theorems $1.3,1.4,1.5]$, in order to study traveling waves and non-trivial long-time behavior, the following assumption is also necessary.

$$
\left(H_{5}\right) \lambda_{P F}(\mathbf{L})>0 .
$$


The collection $\left(H_{1}\right)-\left(H_{5}\right)$ is always assumed from now on. Notice that, although this does not bring any new result, the scalar KPP equation could be seen as a particular KPP system (understanding the pair $\left(H_{1}\right)$ and $\left(H_{5}\right)$ as $r>0$ ). Biological interpretations of these assumptions can be found in [16, Section 1.5].

1.1.2. Traveling waves. Traveling waves are defined in [16] as follows.

Definition. A traveling wave solution of $\left(E_{K P P}\right)$ is a profile-speed pair

$$
(\mathbf{p}, c) \in \mathscr{C}^{2}\left(\mathbb{R}, \mathbb{R}^{N}\right) \times[0,+\infty)
$$

which satisfies:

- $\mathbf{u}:(t, x) \mapsto \mathbf{p}(x-c t)$ is a bounded positive classical solution of $\left(E_{K P P}\right)$;

- $\left(\liminf _{\xi \rightarrow-\infty} p_{i}(\xi)\right)_{i \in[N]}>\mathbf{0}$

- $\lim _{\xi \rightarrow+\infty} \mathbf{p}(\xi)=\mathbf{0}$.

By construction, a traveling wave solution $(\mathbf{p}, c)$ solves $(T W[c])$.

The set of all profiles associated with some speed $c$ is denoted $\mathscr{P}_{c}$. By [16, Theorems 1.5, 1.7], $\mathscr{P}_{c}$ is empty if

$$
c<c^{\star}=\min _{\mu>0} \frac{\lambda_{P F}\left(\mu^{2} \mathbf{D}+\mathbf{L}\right)}{\mu} .
$$

The converse statement (existence of a profile if $c \geq c^{\star}$ ) is likely false in general but is true provided $\mathbf{c}$ is monotonic in the following sense:

$$
D \mathbf{c}(\mathbf{v}) \geq \mathbf{0} \text { for all } \mathbf{v} \in \mathrm{K} .
$$

1.2. Results: at the edge of the fronts. The distribution of the profiles near $+\infty$ follows the "rule of thumb" unfolded in [16]: for several standard problems, KPP systems can be addressed exactly as KPP equations and the results are analogous.

Recall from [16, Lemma 6.2] the notation $\mathbf{n}_{\mu}=\mathbf{n}_{P F}\left(\mu^{2} \mathbf{D}+\mathbf{L}\right)$ for all $\mu \in \mathbb{R}$. Recall also that the equation

$$
\frac{\lambda_{P F}\left(\mu^{2} \mathbf{D}+\mathbf{L}\right)}{\mu}=c
$$

admits no real solution if $c<c^{\star}$, exactly one real solution $\mu_{c^{\star}}>0$ if $c=c^{\star}$ and exactly two real solutions $\mu_{2, c}>\mu_{1, c}>0$ if $c>c^{\star}$. Define subsequently for all $c \geq c^{\star}$ the quantity

$$
\mu_{c}=\min \left\{\mu>0 \mid \frac{\lambda_{P F}\left(\mu^{2} \mathbf{D}+\mathbf{L}\right)}{\mu}=c\right\}= \begin{cases}\mu_{c^{\star}} & \text { if } c=c^{\star} \\ \mu_{1, c} & \text { if } c>c^{\star}\end{cases}
$$

Theorem 1.1. Let

$$
k_{c}= \begin{cases}0 & \text { if } c>c^{\star} \\ 1 & \text { if } c=c^{\star}\end{cases}
$$

For all traveling wave solutions $(\mathbf{p}, c)$, there exists $A>0$ such that, as $\xi \rightarrow+\infty$,

$$
\left\{\begin{array}{c}
\mathbf{p}(\xi) \sim A \xi^{k_{c}} e^{-\mu_{c} \xi} \mathbf{n}_{\mu_{c}} \\
\mathbf{p}^{\prime}(\xi) \sim-\mu_{c} \mathbf{p}(\xi) \\
\mathbf{p}^{\prime \prime}(\xi) \sim \mu_{c}^{2} \mathbf{p}(\xi)
\end{array}\right.
$$


In particular, if $\mathbf{d}=\mathbf{1}_{N, 1}$,

$$
\mathbf{p}(\xi) \sim A \xi^{k_{c}} e^{-\frac{1}{2}\left(c-\sqrt{c^{2}-4 \lambda_{P F}(\mathbf{L})}\right) \xi} \mathbf{n}_{P F}(\mathbf{L}) .
$$

This result is proved in Section 2.

Recall that up to a well-known change of variable $x$, we can always assume without loss of generality $\max _{i \in[N]} d_{i}=1$.

If we have in mind the mutation-competition-diffusion system, then the ecological interpretation of this result is the following: at the leading edge of the invasion, the normalized distribution in phenotypes is $\mathbf{n}_{\mu_{c}}$ and the total population is proportional to $(x-c t)^{k_{c}} \mathrm{e}^{-\mu_{c}(x-c t)}$.

In the special case $c=c^{\star}$, this theorem answers positively a conjecture of Morris, Börger and Crooks [29, Section 4].

Recall that, for the scalar KPP equation, the analogous result on exponential decays has two common proofs, one using ODE arguments and especially phase-plane analysis and the other one using elliptic arguments and especially the comparison principle. Although we could prove the above result by phase-plane analysis indeed, the proof we will provide uses a third technique relying upon the monotonicity of the profiles near $+\infty$, bilateral Laplace transforms and a Ikehara theorem. In our opinion, this technique of proof has independent interest: on one hand, it does not require the comparison principle and, on the other hand, it might be generalizable to non-ODE settings (space-periodic media and pulsating fronts, for instance).

1.3. Results: at the back of the fronts. On the contrary, the distribution of the profiles near $-\infty$ is a much more intricate question, where the multidimensional and non-cooperative structure of the KPP system become preponderant.

Given a positive classical solution $\mathbf{u}$ of $\left(S_{K P P}\right)$, a traveling wave connecting $\mathbf{0}$ to $\mathbf{u}$ is a traveling wave whose profile $\mathbf{p}$ converges to $\mathbf{u}$ as $\xi \rightarrow-\infty$. The general aim is to prove that all traveling waves connect $\mathbf{0}$ to some positive classical solution of $\left(S_{K P P}\right)$ and, when several solutions can be connected to $\mathbf{0}$, to determine somehow which connection prevails. However, as will be explained in Subsection 5.1 (and was first pointed out in Barles-Evans-Souganidis [1]), a general and precise treatment of this problem is likely impossible. It is necessary to focus on special cases. Looking at the literature, we find two frameworks commonly assumed to be mathematically tractable:

- competition terms $c_{i}(\mathbf{v})$ with separated dependencies on $i$ and on $\mathbf{v}$ (Coville-Fabre [6, Dockery-Hutson-Mischaikow-Pernarowski [7], Griette-Raoul [18], Leman-Méléard-Mirrahimi [24]),

- two-component systems with linear competition and vanishingly small mutations (Dockery-Hutson-Mischaikow-Pernarowski [7], Griette-Raoul [18], Morris-Börger-Crooks [29]).

\subsubsection{Separated competition.}

$\left(H_{6}\right)$ There exist $\mathbf{a} \in \mathrm{K}^{++}$and $b: \mathbb{R}^{N} \rightarrow \mathbb{R}$ such that:

- $\mathbf{c}(\mathbf{v})=b(\mathbf{v}) \mathbf{a}$ for all $\mathbf{v} \in \mathrm{K}$;

- the function $w \mapsto b\left(w \mathbf{e}_{i}+\mathbf{v}\right)$ is increasing in $(0,+\infty)$ for all $\mathbf{v} \in \mathbf{K}$ and all $i \in[N]$.

By monotonicity of c, supplementing $\left(H_{1}\right)-\left(H_{5}\right)$ with $\left(H_{6}\right)$ implies the existence of a profile $\mathbf{p} \in \mathscr{P}_{c}$ for all $c \geq c^{\star}$. The decomposition $\mathbf{c}=b \mathbf{a}$ is unique up to 
a multiplicative normalization and we will assume for instance $\max _{i \in[N]} a_{i}=1$. We denote $\mathbf{A}=\operatorname{diag}(\mathbf{a})$ (so that $\mathbf{c}(\mathbf{v}) \circ \mathbf{v}=b(\mathbf{v}) \mathbf{A v})$.

An especially interesting subcase is the intersection between $\left(H_{6}\right)$ and the Lotka-Volterra competition form, where $b$ is a linear functional, that is where there exists $\mathbf{b} \in \mathrm{K}^{++}$ such that

$$
b(\mathbf{v})=\mathbf{b}^{T} \mathbf{v} \text { for all } \mathbf{v} \in \mathrm{K} .
$$

The system $\left(E_{K P P}\right)$ then reads

$$
\partial_{t} \mathbf{u}-\mathbf{D} \partial_{x x} \mathbf{u}=\mathbf{L} \mathbf{u}-\left(\mathbf{b}^{T} \mathbf{u}\right) \mathbf{A} \mathbf{u} .
$$

The systems studied in Dockery-Hutson-Mischaikow-Pernarowski [7] and in Griette-Raoul [18] correspond respectively to

$$
\mathbf{a}=\mathbf{b}=\mathbf{1}_{N, 1}
$$

and to

$$
(\mathbf{a}, \mathbf{b})=\left(\left(\frac{K}{r}, 1\right)^{T}, \frac{r}{K} \mathbf{1}_{2,1}\right) .
$$

The matrix $\mathbf{A}^{-1} \mathbf{L}$ being essentially nonnegative and irreducible, the following eigenpair is well-defined:

$$
\left(\lambda_{\mathbf{a}}, \mathbf{n}_{\mathbf{a}}\right)=\left(\lambda_{P F}\left(\mathbf{A}^{-1} \mathbf{L}\right), \mathbf{n}_{P F}\left(\mathbf{A}^{-1} \mathbf{L}\right)\right) .
$$

Applying [16, Theorem 1.4] to the following two pairs of parameters $(\mathbf{L}, \mathbf{c})$ :

$$
\begin{gathered}
\left(\mathbf{L}, \mathbf{v} \mapsto\left(\mathbf{1}_{1, N} \mathbf{v}\right) \mathbf{a}\right), \\
\left(\mathbf{A}^{-1} \mathbf{L}, \mathbf{v} \mapsto\left(\mathbf{1}_{1, N} \mathbf{v}\right) \mathbf{1}_{N, 1}\right),
\end{gathered}
$$

it is easily deduced that $\lambda_{P F}(\mathbf{L})>0$ if and only if $\lambda_{\mathbf{a}}>0$. By strict monotonicity of $\alpha \mapsto b\left(\alpha \mathbf{n}_{\mathbf{a}}\right)$, we can define $\alpha^{\star}>0$ as the unique solution of $b\left(\alpha \mathbf{n}_{\mathbf{a}}\right)=\lambda_{\mathbf{a}}$. It follows easily that $\mathbf{v}^{\star}=\alpha^{\star} \mathbf{n}_{\mathbf{a}}$ is the unique positive constant solution of $\left(S_{K P P}\right)$. In particular, if $b$ is a linear functional, then

$$
\mathbf{v}^{\star}=\frac{\lambda_{\mathbf{a}}}{\mathbf{b}^{T} \mathbf{n}_{\mathbf{a}}} \mathbf{n}_{\mathbf{a}} .
$$

Theorem 1.2. Assume $\left(H_{6}\right), \mathbf{d}=\mathbf{1}_{N, 1}$ and $\mathbf{a}=\mathbf{1}_{N, 1}$.

For all $c \in\left[c^{\star},+\infty\right)$, let $p_{c} \in \mathscr{C}^{2}(\mathbb{R})$ such that $\left(p_{c}, c\right)$ is the unique traveling wave solution of the scalar equation

$$
\partial_{t} u-\partial_{x x} u=\lambda_{P F}(\mathbf{L}) u-b\left(u \mathbf{n}_{P F}(\mathbf{L})\right) u
$$

connecting 0 to $\alpha^{\star}$ and satisfying $p_{c}(0)=\frac{\alpha^{\star}}{2}$.

Then all $\mathbf{p} \in \mathscr{P}_{c}$ have the form

$$
\mathbf{p}: \xi \mapsto p_{c}\left(\xi-\xi_{0}\right) \mathbf{n}_{P F}(\mathbf{L}) \text { with } \xi_{0} \in \mathbb{R} .
$$

Consequently, $\mathbf{p} \in \mathscr{P}_{c}$ is unique up to translation and connects $\mathbf{0}$ to $\mathbf{v}^{\star}$.

This result is proved in Section 3.2.

This theorem establishes that the set of assumptions $\left(H_{6}\right), \mathbf{d}=\mathbf{1}_{N, 1}, \mathbf{a}=\mathbf{1}_{N, 1}$ is so restrictive that the multidimensional problem can in fact be reduced to the scalar one. This is really the strongest result we could hope for.

Notice that it shows that the following two mutation-competition-diffusion systems:

$$
\partial_{t} \mathbf{u}-\partial_{x x} \mathbf{u}=r \mathbf{u}+\mathbf{M}_{1} \mathbf{u}-\left(\mathbf{b}^{T} \mathbf{u}\right) \mathbf{u}
$$




$$
\partial_{t} \mathbf{u}-\partial_{x x} \mathbf{u}=r \mathbf{u}+\mathbf{M}_{2} \mathbf{u}-\left(\mathbf{b}^{T} \mathbf{u}\right) \mathbf{u}
$$

with $r>0$ and $\mathbf{M}_{1}$ and $\mathbf{M}_{2}$ essentially nonnegative irreducible with null Perron-Frobenius eigenvalues and equal Perron-Frobenius eigenvectors, have the exact same traveling wave solutions. In other words, all else being equal (neutral internal structure), the mutation strategy does not matter. In the absence of mutations, neutral genetic diversity has been studied recently in a collection of papers by Garnier, Hamel, Roques and others (for instance, we refer to [3, 13]). In view of their results on pulled fronts, the preceding theorem indicates that the presence of mutations is a necessary and sufficient condition to ensure the preservation of the genetic diversity during the invasion.

As a side note (slightly off topic), we can use the reduction to the scalar problem to prove the following generalization of a result due to Coville and Fabre [6, Theorem $1.1]$.

Theorem 1.3. Assume $\left(H_{6}\right)$ and $\mathbf{a}=\mathbf{1}_{N, 1}$.

All positive classical solutions of $\left(E_{K P P}^{0}\right)$ set in $(0,+\infty)$ converge as $t \rightarrow+\infty$ to $\mathbf{v}^{\star}$.

Furthermore, if $\mathbf{d}=\mathbf{1}_{N, 1}$, then, for all bounded intervals $I \subset \mathbb{R}$, all bounded positive classical solutions $\mathbf{u}$ of $\left(E_{K P P}\right)$ set in $(0,+\infty) \times \mathbb{R}$ satisfy

$$
\lim _{t \rightarrow+\infty} \sup _{x \in I}\left|\mathbf{u}(t, x)-\mathbf{v}^{\star}\right|=0 \text {. }
$$

Consequently, if $\mathbf{d}=\mathbf{1}_{N, 1}$, the set of bounded nonnegative classical solutions of $\left(S_{K P P}\right)$ is exactly $\left\{\mathbf{0}, \mathbf{v}^{\star}\right\}$.

This result is proved in Section 3.3.

We believe that the preceding two theorems are robust, in that they should remain true in a neighborhood of $(\mathbf{d}, \mathbf{a})=\left(\mathbf{1}_{N, 1}, \mathbf{1}_{N, 1}\right)$. In particular, Theorem 1.2 could be extended by showing with the implicit function theorem that no solution of $(T W[c])$ bifurcates from $\mathbf{v}^{\star}$ at $(\mathbf{d}, \mathbf{a})=\left(\mathbf{1}_{N, 1}, \mathbf{1}_{N, 1}\right)$. Theorem 1.3 could be extended thanks to Conley index theory and a Morse decomposition, exactly as in Dockery-Hutson-Mischaikow-Pernarowski [7, Section 4]. For the sake of brevity, we do not address these questions.

\subsubsection{Two-component systems with linear competition and small mutations.}

$\left(H_{7}\right) \quad N=2$, there exists $\mathbf{C} \gg \mathbf{0}$ such that

$$
\mathbf{c}(\mathbf{v})=\mathbf{C v} \text { for all } \mathbf{v} \in \mathrm{K},
$$

and the vector $\mathbf{r} \in \mathbb{R}^{N}$ given by the unique decomposition of $\mathbf{L}$ of the form

$$
\mathbf{L}=\operatorname{diag}(\mathbf{r})+\mathbf{M} \text { with } \mathbf{1}_{1, N} \mathbf{M}=\mathbf{0}
$$

is positive.

By monotonicity of c, supplementing $\left(H_{1}\right)-\left(H_{5}\right)$ with $\left(H_{7}\right)$ implies the existence of a profile $\mathbf{p} \in \mathscr{P}_{c}$ for all $c \geq c^{\star}$.

When $\left(H_{7}\right)$ is satisfied, we denote $\mathbf{R}=\operatorname{diag}(\mathbf{r})$ and define $(\eta, \mathbf{m}) \in(0,+\infty) \times$ $\mathrm{S}^{++}(\mathbf{0}, 1)$ such that

$$
\mathbf{M}=\eta\left(\begin{array}{cc}
-1 & 1 \\
1 & -1
\end{array}\right) \operatorname{diag}(\mathbf{m}) .
$$

The quantity $\eta$ is unique and commonly referred to as the mutation rate. 
In other words, we are considering the following system:

$$
\left\{\begin{array}{l}
\partial_{t} u_{1}-d_{1} \partial_{x x} u_{1}=r_{1} u_{1}-\left(c_{1,1} u_{1}+c_{1,2} u_{2}\right) u_{1}+\eta m_{1}\left(u_{2}-u_{1}\right) \\
\partial_{t} u_{2}-d_{2} \partial_{x x} u_{2}=r_{2} u_{2}-\left(c_{2,1} u_{1}+c_{2,2} u_{2}\right) u_{2}+\eta m_{2}\left(u_{1}-u_{2}\right)
\end{array}\right.
$$

The idea is to assume that $\eta$ is small compared to $\mathbf{r}$ so that the mutation-competition-diffusion system is close to the pure competition-diffusion system

$$
\left\{\begin{array}{l}
\partial_{t} u_{1}-d_{1} \partial_{x x} u_{1}=r_{1} u_{1}-\left(c_{1,1} u_{1}+c_{1,2} u_{2}\right) u_{1} \\
\partial_{t} u_{2}-d_{2} \partial_{x x} u_{2}=r_{2} u_{2}-\left(c_{2,1} u_{1}+c_{2,2} u_{2}\right) u_{2}
\end{array} . \quad\left(E_{K P P}\right)_{0}\right.
$$

Indeed, two-component competition-diffusion systems being cooperative up to the change of unknowns $v=\frac{r_{2}}{c_{2,2}}-u_{2}$, the maximum principle then simplifies noticeably the characterization of the asymptotic behaviors. In particular, defining $\alpha_{i}=\frac{r_{i}}{c_{i, i}}$ for all $i \in\{1,2\}$ and, if $\operatorname{det} \mathbf{C} \neq 0$,

$$
\mathbf{v}_{m}=\frac{1}{\operatorname{det} \mathbf{C}}\left(\begin{array}{l}
r_{1} c_{2,2}-r_{2} c_{1,2} \\
r_{2} c_{1,1}-r_{1} c_{2,1}
\end{array}\right)
$$

the asymptotic behavior of the solutions of the spatially homogeneous competitive system

$$
\mathbf{u}^{\prime}=\mathbf{R u}-(\mathbf{C u}) \circ \mathbf{u}
$$

is well-known.

(1) [Extinction of $u_{2}$ ] If $\frac{r_{1}}{r_{2}} \geq \max \left(\frac{c_{1,1}}{c_{2,1}}, \frac{c_{1,2}}{c_{2,2}}\right)$ and $\frac{r_{1}}{r_{2}}>\min \left(\frac{c_{1,1}}{c_{2,1}}, \frac{c_{1,2}}{c_{2,2}}\right)$, then $\alpha_{1} \mathbf{e}_{1}$ is globally asymptotically stable in $\mathrm{K}^{++} \cup\left(\operatorname{span}\left(\mathbf{e}_{1}\right) \cap \mathrm{K}^{+}\right)$and $\alpha_{2} \mathbf{e}_{2}$ is globally asymptotically stable in $\operatorname{span}\left(\mathbf{e}_{2}\right) \cap \mathrm{K}^{+}$.

(2) [Coexistence] If $\frac{c_{1,2}}{c_{2,2}}<\frac{r_{1}}{r_{2}}<\frac{c_{1,1}}{c_{2,1}}$, then $\mathbf{v}_{m} \in \mathrm{K}^{++}, \mathbf{v}_{m}$ is globally asymptotically stable in $\mathrm{K}^{++}$and, for all $i \in\{1,2\}, \alpha_{i} \mathbf{e}_{i}$ is globally asymptotically stable in $\operatorname{span}\left(\mathbf{e}_{i}\right) \cap \mathrm{K}^{+}$.

(3) [Competitive exclusion] If $\frac{c_{1,2}}{c_{2,2}}>\frac{r_{1}}{r_{2}}>\frac{c_{1,1}}{c_{2,1}}$, then $\mathbf{v}_{m} \in \mathrm{K}^{++}$and a onedimensional curve $\mathrm{S}$, referred to as the separatrix, induces a partition $\left(\mathrm{K}_{1}^{+}, \mathrm{S}, \mathrm{K}_{2}^{+}\right)$of $\mathrm{K}^{+}$such that $\alpha_{i} \mathbf{e}_{i}$ is globally asymptotically stable in $\mathrm{K}_{i}^{+}$ for all $i \in\{1,2\}$ and $\mathbf{v}_{m}$ is globally asymptotically stable in S.

(4) [Extinction of $u_{1}$ ] If $\frac{r_{1}}{r_{2}} \leq \min \left(\frac{c_{1,1}}{c_{2,1}}, \frac{c_{1,2}}{c_{2,2}}\right)$ and $\frac{r_{1}}{r_{2}}<\max \left(\frac{c_{1,1}}{c_{2,1}}, \frac{c_{1,2}}{c_{2,2}}\right)$, then $\alpha_{2} \mathbf{e}_{2}$ is globally asymptotically stable in $\mathrm{K}^{++} \cup\left(\operatorname{span}\left(\mathbf{e}_{2}\right) \cap \mathrm{K}^{+}\right)$and $\alpha_{1} \mathbf{e}_{1}$ is globally asymptotically stable in $\operatorname{span}\left(\mathbf{e}_{1}\right) \cap \mathrm{K}^{+}$.

The cases 1, 2 and 4 are monostable whereas the case 3 is bistable. The case $\frac{r_{1}}{r_{2}}=\frac{c_{1,1}}{c_{2,1}}=\frac{c_{1,2}}{c_{2,2}}$ is degenerate and is usually discarded.

In the forthcoming statements, $\eta$ is understood as a positive parameter which can be passed to the limit $\eta \rightarrow 0$ (notice that for all $\eta>0,\left(H_{1}\right)-\left(H_{5}\right)$ is satisfied indeed). The system $\left(E_{K P P}\right)$ and the objects $\mathscr{P}_{c}$ and $c^{\star}$ depend on $\eta$ and might be denoted respectively $\left(E_{K P P}\right)_{\eta}, \mathscr{P}_{c, \eta}$ and $c_{\eta}^{\star}$. We define subsequently $\mathscr{E}$ as the set of all $(\eta, \mathbf{p}, c) \in(0,+\infty) \times \mathscr{C}^{2}\left(\mathbb{R}, \mathbb{R}^{2}\right) \times(0,+\infty)$ such that $(\mathbf{p}, c)$ is a traveling wave solution of $\left(E_{K P P}\right)_{\eta}$. Contrarily to the case $\eta>0$, a traveling wave solution of the limiting system $\left(E_{K P P}\right)_{0}$ has no prescribed asymptotic behaviors.

We point out that Morris-Börger-Crooks [29] showed that the limit $c_{0}^{\star}$ of $\left(c_{\eta}^{\star}\right)_{\eta>0}$ as $\eta \rightarrow 0$ is well-defined and satisfies as expected

$$
c_{0}^{\star} \geq 2 \sqrt{\max _{i \in\{1,2\}}\left(d_{i} r_{i}\right)}
$$


with, quite interestingly, strict inequality if

$$
1+\sqrt{1+\frac{\alpha_{i}}{\alpha_{3-i}}}<\frac{2 c_{3-i, 3-i}}{c_{i, 3-i}} \text { and } \frac{d_{i}}{d_{3-i}}+\frac{r_{i}}{r_{3-i}}>2 \text { for all } i \in\{1,2\} .
$$

However, they did not characterize the limiting profiles. This is what we intend to do here (but will only partially achieve).

In the following conjecture, stability is to be understood as local asymptotic stability with respect to $\left(E_{K P P}^{0}\right)$.

Conjecture 1.4. Assume $\left(H_{7}\right)$. Let $\left(\mathbf{p}_{\eta}\right)_{\eta>0}$ and $\left(c_{\eta}\right)_{\eta \geq 0}$ such that

$$
\left\{\begin{array}{c}
\left(\eta, \mathbf{p}_{\eta}, c_{\eta}\right) \in \mathscr{E} \quad \text { for all } \eta>0, \\
c_{0}=\lim _{\eta \rightarrow 0} c_{\eta} .
\end{array}\right.
$$

i) Assume that both $\alpha_{1} \mathbf{e}_{1}$ and $\alpha_{2} \mathbf{e}_{2}$ are stable and that $c_{\alpha_{1} \mathbf{e}_{1} \rightarrow \alpha_{2} \mathbf{e}_{2}} \neq 0$. Then there exists $\left(\xi_{\eta}\right)_{\eta>0}$ such that $\left(\xi \mapsto \mathbf{p}_{\eta}\left(\xi+\xi_{\eta}\right), c_{\eta}\right)_{\eta>0}$ converges in $\left(\mathscr{C}_{\text {loc }}^{2}\left(\mathbb{R}, \mathbb{R}^{2}\right) \cap \mathscr{L}^{\infty}\left(\mathbb{R}, \mathbb{R}^{2}\right)\right) \times \mathbb{R}$ as $\eta \rightarrow 0$ to a semi-extinct traveling wave solution $\left(p_{0} \mathbf{e}_{i}, c_{0}\right)$ of $\left(E_{K P P}\right)_{0}$ connecting $\mathbf{0}$ to $\alpha_{i} \mathbf{e}_{i}$ with

$$
i= \begin{cases}1 & \text { if } c_{\alpha_{1} \mathbf{e}_{1} \rightarrow \alpha_{2} \mathbf{e}_{2}}>0, \\ 2 & \text { if } c_{\alpha_{1} \mathbf{e}_{1} \rightarrow \alpha_{2} \mathbf{e}_{2}}<0 .\end{cases}
$$

ii) Assume that there is a unique stable state $\mathbf{v}_{s} \in\left\{\alpha_{1} \mathbf{e}_{1}, \alpha_{2} \mathbf{e}_{2}, \mathbf{v}_{m}\right\}$. Then one and only one of the following two properties holds true.

(a) There exists $\left(\xi_{\eta}\right)_{\eta>0}$ such that $\left(\xi \mapsto \mathbf{p}_{\eta}\left(\xi+\xi_{\eta}\right), c_{\eta}\right)_{\eta>0}$ converges in $\left(\mathscr{C}_{\text {loc }}^{2}\left(\mathbb{R}, \mathbb{R}^{2}\right) \cap \mathscr{L}^{\infty}\left(\mathbb{R}, \mathbb{R}^{2}\right)\right) \times \mathbb{R}$ as $\eta \rightarrow 0$ to a component-wise monotonic traveling wave solution $\left(\mathbf{p}_{0}, c_{0}\right)$ of $\left(E_{K P P}\right)_{0}$ connecting $\mathbf{0}$ to $\mathbf{v}_{s}$.

(b) There exist $\left(\xi_{\eta}^{1}\right)_{\eta>0},\left(\xi_{\eta}^{2}\right)_{\eta>0}$ and a unique $i \in \mathrm{I}_{u}$ such that, as $\eta \rightarrow 0$ :

- $\xi_{\eta}^{2}-\xi_{\eta}^{1} \rightarrow+\infty$

- $\left(\xi \mapsto \mathbf{p}_{\eta}\left(\xi+\xi_{\eta}^{2}\right), c_{\eta}\right)_{\eta>0}$ converges in $\mathscr{C}_{\text {loc }}^{2}\left(\mathbb{R}, \mathbb{R}^{2}\right) \times \mathbb{R}$ to a semiextinct traveling wave solution $\left(p_{\text {front }} \mathbf{e}_{i}, c_{0}\right)$ of $\left(E_{K P P}\right)_{0}$ connecting $\mathbf{0}$ to $\alpha_{i} \mathbf{e}_{i}$;

- $\left(\xi \mapsto \mathbf{p}_{\eta}\left(\xi+\xi_{\eta}^{1}\right), c_{\eta}\right)_{\eta>0}$ converges in $\mathscr{C}_{\text {loc }}^{2}\left(\mathbb{R}, \mathbb{R}^{2}\right) \times \mathbb{R}$ to a componentwise monotonic traveling wave solution $\left(\mathbf{p}_{\text {back }}, c_{0}\right)$ of $\left(E_{K P P}\right)_{0}$ connecting $\alpha_{i} \mathbf{e}_{i}$ to $\mathbf{v}_{s}$.

We emphasize once more that traveling waves with minimal speed $c_{\eta}^{\star}$ do not, in general, converge to a traveling wave with minimal speed. In particular, Figure 5.1 illustrates an interesting case of invasion driven by the fast phenotype $u_{2}$ but where the only settler is the slow phenotype $u_{1}$. This is reminiscent of Griette-Raoul [18], where an analogous result was established analytically under a stronger scaling.

Conjecture 1.4, i) is expected to be a very difficult problem and seems to be beyond our reach. We leave it as an open problem.

On the contrary, regarding Conjecture 1.4 ii) a partial confirmation is within reach. On one hand, we point out that the special case

$$
\frac{c_{1,1}}{c_{2,1}}=\frac{c_{1,2}}{c_{2,2}}=1 \text { and } \mathbf{d}=\mathbf{1}_{2,1}
$$

is somehow solved by Theorem 1.2 without any assumption on $\mathbf{r}$. On the other hand, we also have the following general theorem which concerns all monostable 
cases apart from

$$
\begin{aligned}
& \frac{c_{1,1}}{c_{2,1}}<\frac{c_{1,2}}{c_{2,2}}=\frac{r_{1}}{r_{2}}, \\
& \frac{r_{1}}{r_{2}}=\frac{c_{1,1}}{c_{2,1}}<\frac{c_{1,2}}{c_{2,2}} .
\end{aligned}
$$

Theorem 1.5. Assume $\left(H_{7}\right)$ and the existence of $i \in\{1,2\}$ such that

$$
\frac{r_{i}}{r_{3-i}}>\frac{c_{i, 3-i}}{c_{3-i, 3-i}}
$$

Let

$$
\mathbf{v}_{s}=\left\{\begin{array}{cl}
\alpha_{i} \mathbf{e}_{i} & \text { if } \frac{r_{i}}{r_{3-i}} \geq \frac{c_{i, i}}{c_{3-i, i}} \\
\mathbf{v}_{m} & \text { if } \frac{r_{i}}{r_{3-i}}<\frac{c_{i, i}}{c_{3-i, i}}
\end{array}\right.
$$

For all $\left(\mathbf{p}_{\eta}\right)_{\eta>0}$ and $\left(c_{\eta}\right)_{\eta \geq 0}$ such that

$$
\left\{\begin{array}{c}
\left(\eta, \mathbf{p}_{\eta}, c_{\eta}\right) \in \mathscr{E} \quad \text { for all } \eta>0, \\
c_{0}=\lim _{\eta \rightarrow 0} c_{\eta}
\end{array}\right.
$$

there exists $\left(\zeta_{\eta}\right)_{\eta>0}$ such that, as $\eta \rightarrow 0,\left(\xi \mapsto \mathbf{p}_{\eta}\left(\xi+\zeta_{\eta}\right), c_{\eta}\right)_{\eta>0}$ converges up to extraction in $\mathscr{C}_{\text {loc }}^{2}\left(\mathbb{R}, \mathbb{R}^{2}\right) \times \mathbb{R}$ to a traveling wave solution $\left(\mathbf{p}, c_{0}\right)$ of $\left(E_{K P P}\right)_{0}$ achieving one of the following connections:

(1) $\mathbf{0}$ to $\mathbf{v}_{s}$,

(2) $\alpha_{3-i} \mathbf{e}_{3-i}$ to $\mathbf{v}_{s}$,

(3) $\mathbf{0}$ to $\alpha_{i} \mathbf{e}_{i}$ with $\mathbf{p}$ semi-extinct.

This result is proved in Section 4.

Let us clarify how this result confirms partially Conjecture 1.4 , ii) and what are the remaining open questions.

- Assume $\mathbf{v}_{s}=\mathbf{v}_{m}$. Up to the component-wise monotonicity of the profile in the first and second cases, the three connections above correspond exactly to the three possible limiting profiles of Conjecture 1.4 ii). Moreover we can apply the theorem with $i=1$ and $i=2$ and obtain two limiting profiles. However, at this point, the normalizations $\left(\zeta_{\eta}^{1}\right)_{\eta>0}$ and $\left(\zeta_{\eta}^{2}\right)_{\eta>0}$ are unrelated and nine possible pairs of profiles seem to exist. We do not know how to prove that only the three following situations actually occur: $\mathbf{0}$ to $\mathbf{v}_{m}$ and $\mathbf{0}$ to $\mathbf{v}_{m}$ with $\left(\zeta_{\eta}^{2}-\zeta_{\eta}^{1}\right)_{\eta>0}$ bounded, semi-extinct $\mathbf{0}$ to $\alpha_{1} \mathbf{e}_{1}$ and $\alpha_{1} \mathbf{e}_{1}$ to $\mathbf{v}_{m}$ with $\zeta_{\eta}^{2}-\zeta_{\eta}^{1} \rightarrow-\infty$, semi-extinct $\mathbf{0}$ to $\alpha_{2} \mathbf{e}_{2}$ and $\alpha_{2} \mathbf{e}_{2}$ to $\mathbf{v}_{m}$ with $\zeta_{\eta}^{2}-\zeta_{\eta}^{1} \rightarrow+\infty$

- Assume $\mathbf{v}_{s}=\alpha_{i} \mathbf{e}_{i}$. The third connection above is actually a subcase of the first one and the normalization $\left(\zeta_{\eta}\right)_{\eta>0}$ is unable to track the semiextinct limiting profile connecting $\mathbf{0}$ to $\alpha_{3-i} \mathbf{e}_{3-i}$. This is not a question of optimality of the proof: the normalization $\left(\zeta_{\eta}\right)_{\eta>0}$ is precisely chosen so that $p_{i}$ is always non-zero. Hence $\left(\zeta_{\eta}\right)_{\eta>0}$ corresponds either to $\left(\xi_{\eta}\right)_{\eta>0}$ or to $\left(\xi_{\eta}^{1}\right)_{\eta>0}$. The construction of the normalization $\left(\xi_{\eta}^{2}\right)_{\eta>0}$ of Conjecture 1.4 . ii) is a completely open problem. Of course, once this problem is solved, it remains to relate the limiting profiles and the normalizations, as in the case $\mathbf{v}_{s}=\mathbf{v}_{m}$. 


\section{THE EDGE OF THE FRONTS}

In this section, we fix a traveling wave $(\mathbf{p}, c)$ and we prove Theorem 1.1 .

\subsection{Preparatory lemmas and the Ikehara theorem.}

Lemma 2.1. For all $i \in[N]$,

$$
\begin{gathered}
\left\{\liminf _{+\infty} \frac{-p_{i}^{\prime}}{p_{i}}, \limsup _{+\infty} \frac{-p_{i}^{\prime}}{p_{i}}\right\} \subset\left\{\mu \in(0,+\infty) \mid \frac{\lambda_{P F}\left(\mu^{2} \mathbf{D}+\mathbf{L}\right)}{\mu}=c\right\}, \\
\left\{\underset{+\infty}{\liminf } \frac{p_{i}^{\prime \prime}}{p_{i}}, \limsup _{+\infty} \frac{p_{i}^{\prime \prime}}{p_{i}}\right\} \subset\left\{\mu^{2} \in(0,+\infty) \mid \frac{\lambda_{P F}\left(\mu^{2} \mathbf{D}+\mathbf{L}\right)}{\mu}=c\right\} .
\end{gathered}
$$

Consequently, there exists $\tilde{\xi} \in \mathbb{R}$ such that $\mathbf{p}$ is component-wise strictly convex in $[\tilde{\xi},+\infty)$.

Proof. The proof of

$$
\min _{i \in[N]} \liminf _{+\infty} \frac{-p_{i}^{\prime}}{p_{i}} \in\left\{\mu>0 \mid \frac{\lambda_{P F}\left(\mu^{2} \mathbf{D}+\mathbf{L}\right)}{\mu}=c\right\}
$$

can be found in [16, Proposition 6.10]. The proof also directly yields that for any sequence $\left(\xi_{n}\right)_{n \in \mathbb{N}}$ such that $\xi_{n} \rightarrow+\infty$ and such that there exists $j \in[N]$ satisfying

$$
\lim _{n \rightarrow+\infty} \frac{-p_{j}^{\prime}\left(\xi_{n}\right)}{p_{j}\left(\xi_{n}\right)}=\min _{i \in[N]} \liminf _{+\infty} \frac{-p_{i}^{\prime}}{p_{i}},
$$

convergence occurs in the following sense:

$$
\lim _{n \rightarrow+\infty}\left(\frac{-p_{i}^{\prime}\left(\xi_{n}\right)}{p_{i}\left(\xi_{n}\right)}\right)_{i \in[N]}=\left(\min _{i \in[N]} \liminf _{+\infty} \frac{-p_{i}^{\prime}}{p_{i}}\right) \mathbf{1}_{N, 1} .
$$

The proof of

$$
\max _{i \in[N]} \limsup _{+\infty} \frac{-p_{i}^{\prime}}{p_{i}} \in\left\{\mu>0 \mid \frac{\lambda_{P F}\left(\mu^{2} \mathbf{D}+\mathbf{L}\right)}{\mu}=c\right\}
$$

is a slight modification of the preceding proof, where the quantity

$$
\bar{\Lambda}=\max _{i \in[N]} \limsup _{\xi \rightarrow+\infty} \frac{p_{i}^{\prime}(\xi)}{p_{i}(\xi)}
$$

is replaced by

$$
\underline{\Lambda}=\min _{i \in[N]} \liminf _{\xi \rightarrow+\infty} \frac{p_{i}^{\prime}(\xi)}{p_{i}(\xi)} .
$$

Similarly, we also obtain directly that for any sequence $\left(\xi_{n}\right)_{n \in \mathbb{N}}$ such that $\xi_{n} \rightarrow+\infty$ and such that there exists $j \in[N]$ satisfying

$$
\lim _{n \rightarrow+\infty} \frac{-p_{j}^{\prime}\left(\xi_{n}\right)}{p_{j}\left(\xi_{n}\right)}=\max _{i \in[N]} \limsup _{+\infty} \frac{-p_{i}^{\prime}}{p_{i}}
$$

convergence occurs in the following sense:

$$
\lim _{n \rightarrow+\infty}\left(\frac{-p_{i}^{\prime}\left(\xi_{n}\right)}{p_{i}\left(\xi_{n}\right)}\right)_{i \in[N]}=\left(\max _{i \in[N]} \limsup _{+\infty} \frac{-p_{i}^{\prime}}{p_{i}}\right) \mathbf{1}_{N, 1} .
$$


The statements regarding $\left(\frac{p_{i}^{\prime \prime}}{p_{i}}\right)_{i \in[N]}$ are again established very similarly. The quantity

$$
\bar{\Lambda}=\max _{i \in[N]} \limsup _{\xi \rightarrow+\infty} \frac{p_{i}^{\prime}(\xi)}{p_{i}(\xi)}
$$

is replaced by

$$
\underline{\Theta}=\min _{i \in[N]} \liminf _{\xi \rightarrow+\infty} \frac{p_{i}^{\prime \prime}(\xi)}{p_{i}(\xi)}
$$

and

$$
\bar{\Theta}=\max _{i \in[N]} \liminf _{\xi \rightarrow+\infty} \frac{p_{i}^{\prime \prime}(\xi)}{p_{i}(\xi)}
$$

respectively, and the function

$$
\mathbf{w}_{n}=\bar{\Lambda} \hat{\mathbf{p}}_{n}-\hat{\mathbf{p}}_{n}^{\prime}
$$

is replaced by

$$
\mathbf{w}_{n}=\underline{\Theta} \hat{\mathbf{p}}_{n}-\hat{\mathbf{p}}_{n}^{\prime \prime}
$$

and

$$
\mathbf{w}_{n}=\bar{\Theta} \hat{\mathbf{p}}_{n}-\hat{\mathbf{p}}_{n}^{\prime \prime}
$$

respectively. Since $\hat{\mathbf{p}}_{\infty}$ is nonnegative nonzero and $\mathbf{w}_{\infty}=\mathbf{0}$, necessarily $\underline{\Theta}>0$ and $\bar{\Theta}>0$ and then, as in [16. Proposition 6.10], both quantities have the form $\mu^{2}$ with $\mu$ solution of $\frac{\lambda_{P F}\left(\mu^{2} \mathbf{D}+\mathbf{L}\right)}{\mu}=c$.

Finally, the strict convexity in a neighborhood of $+\infty$ is deduced exactly as the monotonicity in the proof of [16, Proposition 6.10].

We will also need the Ikehara theorem [4, Proposition 2.3], commonly used in such problems (see for instance Guo-Wu [20]), as well as a lemma due to Volpert, Volpert and Volpert [33, Chapter 5, Lemma 4.1].

Theorem 2.2. [Ikehara] Let $f:(0,+\infty) \rightarrow(0,+\infty)$ be a decreasing function. Assume that there exist $\bar{\lambda} \in(0,+\infty), k \in(-1,+\infty)$ and an analytic function

$$
h:(0, \bar{\lambda}]+i \mathbb{R} \rightarrow(0,+\infty)
$$

such that

$$
\int_{0}^{+\infty} e^{\lambda x} f(x) d x=\frac{h(\lambda)}{(\bar{\lambda}-\lambda)^{k+1}} \text { for all } \lambda \in(0, \bar{\lambda}) .
$$

Then

$$
\lim _{x \rightarrow+\infty} f(x) \frac{e^{\bar{\lambda} x}}{x^{k}}=\frac{h(\bar{\lambda})}{\Gamma(\bar{\lambda}+1)} .
$$

Lemma 2.3. [Volpert-Volpert-Volpert] Let $\mathbf{A}$ be an essentially nonnegative matrix and let $\mathbf{z} \in \mathbb{C}^{N}$.

If

$$
\left\{\begin{array}{c}
s p \mathbf{A} \subset(-\infty, 0)+i \mathbb{R} \\
\left(\operatorname{Re}\left(z_{k}\right)\right)_{k \in[N]} \leq \mathbf{0},
\end{array}\right.
$$

then

$$
s p(\mathbf{A}+\operatorname{diag}(\mathbf{z})) \subset(-\infty, 0)+i \mathbb{R} .
$$


2.2. Convergence at the edge. Let

$$
k_{c}= \begin{cases}0 & \text { if } c>c^{\star} \\ 1 & \text { if } c=c^{\star}\end{cases}
$$

Proposition 2.4. There exists $A>0$ such that, as $\xi \rightarrow+\infty$,

$$
\left\{\begin{array}{c}
\mathbf{p}(\xi) \sim A \xi^{k_{c}} e^{-\mu_{c} \xi} \mathbf{n}_{\mu_{c}}, \\
\mathbf{p}^{\prime}(\xi) \sim-\mu_{c} \mathbf{p}(\xi) \\
\mathbf{p}^{\prime \prime}(\xi) \sim \mu_{c}^{2} \mathbf{p}(\xi)
\end{array}\right.
$$

Proof. Fix temporarily $\mu \in\left(0, \mu_{c}\right)+i \mathbb{R}$. In view of Lemma 2.1 and of the Gronwall lemma,

$$
\begin{gathered}
\xi \mapsto \mathrm{e}^{\mu \xi} \mathbf{p}(\xi) \in \mathscr{L}^{1}\left(\mathbb{R}, \mathbb{C}^{N}\right), \\
\xi \mapsto \mathrm{e}^{\mu \xi} \mathbf{c}(\mathbf{p}(\xi)) \circ \mathbf{p}(\xi) \in \mathscr{L}^{1}\left(\mathbb{R}, \mathbb{C}^{N}\right) .
\end{gathered}
$$

Multiplying $(T W[c])$ by $\mathrm{e}^{\mu \xi}$, integrating by parts over $\mathbb{R}$ and defining

we get easily

$$
\begin{gathered}
\mathbf{f}_{+}(\mu)=\int_{0}^{+\infty} \mathrm{e}^{\mu \xi} \mathbf{p}(\xi) \mathrm{d} \xi \\
\mathbf{f}_{-}(\mu)=\int_{-\infty}^{0} \mathrm{e}^{\mu \xi} \mathbf{p}(\xi) \mathrm{d} \xi \\
\mathbf{f}_{\mathbf{c}}(\mu)=\int_{\mathbb{R}} \mathrm{e}^{\mu \xi} \mathbf{c}(\mathbf{p}(\xi)) \circ \mathbf{p}(\xi) \mathrm{d} \xi
\end{gathered}
$$

$$
\left(\mu^{2} \mathbf{D}-c \mu \mathbf{I}+\mathbf{L}\right)\left(\mathbf{f}_{+}(\mu)+\mathbf{f}_{-}(\mu)\right)=\mathbf{f}_{\mathbf{c}}(\mu),
$$

whence, denoting $\operatorname{adj}\left(\mu^{2} \mathbf{D}-c \mu \mathbf{I}+\mathbf{L}\right)$ the adjugate matrix of $\mu^{2} \mathbf{D}-c \mu \mathbf{I}+\mathbf{L}$, we find

$\operatorname{det}\left(\mu^{2} \mathbf{D}-c \mu \mathbf{I}+\mathbf{L}\right) \mathbf{f}_{+}(\mu)=\operatorname{adj}\left(\mu^{2} \mathbf{D}-c \mu \mathbf{I}+\mathbf{L}\right) \mathbf{f}_{\mathbf{c}}(\mu)-\operatorname{det}\left(\mu^{2} \mathbf{D}-c \mu \mathbf{I}+\mathbf{L}\right) \mathbf{f}_{-}(\mu)$.

The functions $\mathbf{f}_{+}, \mathbf{f}_{-}$and $\mathbf{f}_{\mathbf{c}}$ defined above are respectively analytic in $\left(0, \mu_{c}\right)+i \mathbb{R}$, $(0,+\infty)+i \mathbb{R}$ and $\left(0,2 \mu_{c}\right)+i \mathbb{R}$ (by local Lipschitz-continuity of $\mathbf{c},\left(H_{2}\right)$ and global boundedness of $\mathbf{p})$.

The function

$$
\begin{array}{llc}
\mathbb{C} & \rightarrow & \mathbb{C} \\
\mu & \mapsto & \operatorname{det}\left(\mu^{2} \mathbf{D}-c \mu \mathbf{I}+\mathbf{L}\right)
\end{array}
$$

is polynomial (whence analytic). Let $\mathbf{Z} \subset \mathbb{C}$ be the finite set of its roots, counted with algebraic multiplicity. In particular, $\mu_{c} \in \mathbf{Z}$ with multiplicity $k_{c}+1$.

For all $\mu \in\left(\left(0, \mu_{c}\right)+i \mathbb{R}\right) \backslash \mathrm{Z}$,

$$
\mathbf{f}_{+}(\mu)=\left(\mu^{2} \mathbf{D}-c \mu \mathbf{I}+\mathbf{L}\right)^{-1} \mathbf{f}_{\mathbf{c}}(\mu)-\mathbf{f}_{-}(\mu) .
$$

The function

$$
\mu \mapsto\left(\mu^{2} \mathbf{D}-c \mu \mathbf{I}+\mathbf{L}\right)^{-1} \mathbf{f}_{\mathbf{c}}(\mu)
$$

is well-defined and analytic in $\left(\left(0, \mu_{c}\right)+i \mathbb{R}\right) \backslash \mathbf{Z}$, where it coincides with $\mathbf{f}_{+}+\mathbf{f}_{-}$ which is analytic in $\left(0, \mu_{c}\right)+i \mathbb{R}$.

Define the analytic function

$$
\begin{array}{clc}
\mathbf{h}:\left(0, \mu_{c}\right)+i \mathbb{R} & \rightarrow & \mathbb{R}^{N} \\
\mu & \mapsto & \left(\mu_{c}-\mu\right)^{k_{c}+1} \mathbf{f}_{+}(\mu)
\end{array}
$$

so that

$$
\mathbf{f}_{+}(\mu)=\frac{\mathbf{h}(\mu)}{\left(\mu_{c}-\mu\right)^{k_{c}+1}} \text { for all } \mu \in\left(0, \mu_{c}\right)+i \mathbb{R} .
$$


Since, for all $\mu \in\left(\left(0, \mu_{c}\right)+i \mathbb{R}\right) \backslash Z$,

$$
\mathbf{h}(\mu)=\frac{\left(\mu_{c}-\mu\right)^{k_{c}+1}}{\operatorname{det}\left(\mu^{2} \mathbf{D}-c \mu \mathbf{I}+\mathbf{L}\right)} \operatorname{adj}\left(\mu^{2} \mathbf{D}-c \mu \mathbf{I}+\mathbf{L}\right) \mathbf{f}_{\mathbf{c}}(\mu)-\left(\mu_{c}-\mu\right)^{k_{c}+1} \mathbf{f}_{-}(\mu)
$$

the function $\mathbf{h}$ can be analytically extended on $\left(0, \mu_{c}\right]+i \mathbb{R}$ if and only if

$$
\mu \mapsto \frac{\left(\mu_{c}-\mu\right)^{k_{c}+1}}{\operatorname{det}\left(\mu^{2} \mathbf{D}-c \mu \mathbf{I}+\mathbf{L}\right)}
$$

has no pole in $\left\{\mu_{c}\right\}+i \mathbb{R}$.

Let $\theta \in \mathbb{R} \backslash\{0\}$. In view of

$$
\left(\mu_{c}+i \theta\right)^{2} \mathbf{D}-c\left(\mu_{c}+i \theta\right) \mathbf{I}+\mathbf{L}=\mu_{c}^{2} \mathbf{D}-c \mu_{c} \mathbf{I}+\mathbf{L}-\theta^{2} \mathbf{D}+i \theta\left(2 \mu_{c} \mathbf{D}-c \mathbf{I}\right)
$$

and

$$
\begin{aligned}
\lambda_{P F}\left(\mu_{c}^{2} \mathbf{D}-c \mu_{c} \mathbf{I}+\mathbf{L}-\theta^{2} \mathbf{D}\right) & \leq \lambda_{P F}\left(\mu_{c}^{2} \mathbf{D}-c \mu_{c} \mathbf{I}+\mathbf{L}-\theta^{2} \min _{k \in[N]} d_{k}\right) \\
& =\lambda_{P F}\left(\mu_{c}^{2} \mathbf{D}-c \mu_{c} \mathbf{I}+\mathbf{L}\right)-\theta^{2} \min _{k \in[N]} d_{k} \\
& =-\theta^{2} \min _{k \in[N]} d_{k}
\end{aligned}
$$

Lemma 2.3 yields that

$$
\operatorname{sp}\left(\left(\mu_{c}^{2} \mathbf{D}-c \mu_{c} \mathbf{I}+\mathbf{L}-\theta^{2} \mathbf{D}\right)+\operatorname{diag}\left(i \theta\left(2 \mu_{c} d_{k}-c\right)\right)_{k \in[N]}\right) \subset(-\infty, 0)+i \mathbb{R} .
$$

Hence $\mu \mapsto \frac{\left(\mu_{c}-\mu\right)^{k_{c}+1}}{\operatorname{det}\left(\mu^{2} \mathbf{D}-c \mu \mathbf{I}+\mathbf{L}\right)}$ has no pole in $\left\{\mu_{c}\right\}+i(\mathbb{R} \backslash\{0\})$ and then it has no pole in $\left\{\mu_{c}\right\}+i \mathbb{R}$ indeed.

We are now in position to apply the Ikehara theorem component-wise and to deduce from it the existence of $\mathbf{n} \in \mathrm{S}^{+}(\mathbf{0}, 1)$ and $A \geq 0$ such that

$$
\lim _{\xi \rightarrow+\infty} \mathbf{p}(\xi) \frac{\mathrm{e}^{\mu_{c} \xi}}{\xi^{k_{c}}}=A \mathbf{n} .
$$

In particular, for all $k \in[N]$ such that $n_{k}>0$,

$$
\lim _{\zeta \rightarrow+\infty} \frac{\mathbf{p}(\xi+\zeta)}{p_{k}(\zeta)} \mathrm{e}^{\mu_{c} \xi}=\frac{1}{n_{k}} \mathbf{n} .
$$

However, back to the proof of Lemma 2.1. there exists $k \in[N]$ and a sequence $\left(\xi_{n}\right)_{n \in \mathbb{N}}$ such that $\xi_{n} \rightarrow+\infty,\left(\frac{-p_{k}^{\prime}\left(\xi_{n}\right)}{p_{k}\left(\xi_{n}\right)}\right)_{n \in \mathbb{N}}$ converges to

$$
\mu=\max _{k \in[N]} \limsup _{+\infty} \frac{-p_{k}^{\prime}}{p_{k}},
$$

and

converges in $\mathscr{C}_{\text {loc }}^{2}$ to

$$
\left(\xi \mapsto \frac{\mathbf{p}\left(\xi+\zeta_{n}\right)}{p_{k}\left(\zeta_{n}\right)}\right)_{n \in \mathbb{N}}
$$

$$
\xi \mapsto \frac{1}{n_{\mu, k}} \mathrm{e}^{-\mu \xi} \mathbf{n}_{\mu} .
$$

This clearly implies $\mu=\mu_{c}$ and $\mathbf{n}=\mathbf{n}_{\mu_{c}}$.

Consequently, $A>0$,

$$
\lim _{\xi \rightarrow+\infty} \mathbf{p}(\xi) \frac{\mathrm{e}^{\mu_{c} \xi}}{\xi^{k_{c}}}=A \mathbf{n}_{\mu_{c}}
$$


and, by Lemma 2.1

$$
\mu_{c} \leq \min _{k \in[N]} \liminf _{+\infty} \frac{-p_{k}^{\prime}}{p_{k}} \leq \max _{k \in[N]} \limsup _{+\infty} \frac{-p_{k}^{\prime}}{p_{k}}=\mu_{c},
$$

that is

$$
\lim _{+\infty}\left(\frac{-p_{k}^{\prime}}{p_{k}}\right)_{k \in[N]}=\mu_{c}
$$

Quite similarly, we also obtain

$$
\lim _{+\infty}\left(\frac{p_{k}^{\prime \prime}}{p_{k}}\right)_{k \in[N]}=\mu_{c}^{2}
$$

If $\mathbf{d}=\mathbf{1}_{N, 1}$, the quantities at hand are:

$$
\begin{aligned}
\left(\mu_{c}, \mathbf{n}_{\mu_{c}}\right) & =\left(\min \left\{\mu>0 \mid \lambda_{P F}\left(\mu^{2} \mathbf{I}+\mathbf{L}\right)=c \mu\right\}, \mathbf{n}_{P F}\left(\mu_{c}^{2} \mathbf{I}+\mathbf{L}\right)\right) \\
& =\left(\frac{1}{2}\left(c-\sqrt{c^{2}-4 \lambda_{P F}(\mathbf{L})}\right), \mathbf{n}_{P F}(\mathbf{L})\right)
\end{aligned}
$$

and an obvious corollary follows.

\section{THE BACK OF THE FRONTS: SEPARATED COMPETITION}

In this section, we assume $\left(H_{6}\right)$ and $\mathbf{a}=\mathbf{1}_{N, 1}$ and prove Theorem 1.2 and Theorem 1.3 .

3.1. Main tools: Jordan normal form and Perron-Frobenius projection. Let $m \in[N]$ be the number of pairwise distinct eigenvalues of $\mathbf{L}\left(\lambda_{P F}(\mathbf{L})\right.$ being simple, $m \geq 2)$ and let $\left(\lambda_{k}\right)_{k \in[m]} \in \mathbb{C}^{m}$ be the pairwise distinct complex eigenvalues of $\mathbf{L}$ ordered so that $\left(\operatorname{Re}\left(\lambda_{k}\right)\right)_{k \in[m]}$ is a nondecreasing family (in particular, $\lambda_{m}=$ $\lambda_{P F}(\mathbf{L})$ and $\left.\operatorname{Re}\left(\lambda_{m-1}\right)<\lambda_{P F}(\mathbf{L})\right)$.

Let $\mathbf{P} \in \mathrm{GL}(\mathbb{C})$ be such that $\mathbf{J}=\mathbf{P L P} \mathbf{P}^{-1}$ is the Jordan normal form of $\mathbf{L}$ :

$$
\mathbf{J}=\left(\begin{array}{cccc}
\lambda_{P F}(\mathbf{L}) & \mathbf{0} & \cdots & \mathbf{0} \\
\mathbf{0} & \mathbf{J}_{m-1} & \ddots & \vdots \\
\vdots & \ddots & \ddots & \mathbf{0} \\
\mathbf{0} & \cdots & \mathbf{0} & \mathbf{J}_{1}
\end{array}\right)
$$

where, for all $k \in[m-1], \mathbf{J}_{k}$ is the (upper triangular) Jordan block associated with the eigenvalue $\lambda_{k}$.

Noticing that

$$
\begin{gathered}
\mathbf{L} \mathbf{P}^{-1} \mathbf{e}_{1}=\mathbf{P}^{-1} \mathbf{J} \mathbf{e}_{1}=\lambda_{P F}(\mathbf{L}) \mathbf{P}^{-1} \mathbf{e}_{1}, \\
\mathbf{e}_{1}^{T} \mathbf{P L}=\mathbf{e}_{1}^{T} \mathbf{J P}=\lambda_{P F}(\mathbf{L}) \mathbf{e}_{1}^{T} \mathbf{P},
\end{gathered}
$$

it follows that $\mathbf{P}^{-1} \mathbf{e}_{1} \in \operatorname{spann}_{P F}(\mathbf{L})$ and $\mathbf{e}_{1}^{T} \mathbf{P} \in \operatorname{spann}_{P F}\left(\mathbf{L}^{T}\right)^{T}$. In particular, we can normalize without loss of generality $\mathbf{P}$ so that $\mathbf{P}^{-1} \mathbf{e}_{1}=\mathbf{n}_{P F}(\mathbf{L})$ and then deduce from $\mathbf{e}_{1}^{T} \mathbf{P n}_{P F}(\mathbf{L})=1$ that

$$
\mathbf{e}_{1}^{T} \mathbf{P}=\frac{1}{\mathbf{n}_{P F}\left(\mathbf{L}^{T}\right)^{T} \mathbf{n}_{P F}(\mathbf{L})} \mathbf{n}_{P F}\left(\mathbf{L}^{T}\right)^{T} .
$$


From the preceding equality, it follows directly that the Perron-Frobenius projection, defined as

satisfies

$$
\boldsymbol{\Pi}_{P F}(\mathbf{L})=\frac{\mathbf{n}_{P F}(\mathbf{L}) \mathbf{n}_{P F}\left(\mathbf{L}^{T}\right)^{T}}{\mathbf{n}_{P F}\left(\mathbf{L}^{T}\right)^{T} \mathbf{n}_{P F}(\mathbf{L})},
$$

$$
\mathbf{P} \Pi_{P F}(\mathbf{L}) \mathbf{P}^{-1}=\operatorname{diag}\left(\mathbf{e}_{1}\right) .
$$

3.2. Uniqueness up to translation of the profile. In this subsection, we assume $\mathbf{d}=\mathbf{1}_{N, 1}$, we fix $c \geq c^{\star}$ and we prove Theorem 1.2. The scalar front $p_{c}$ is defined as in the statement of the theorem.

Proposition 3.1. All $\mathbf{p} \in \mathscr{P}_{c}$ have the form

$$
\mathbf{p}: \xi \mapsto p_{c}\left(\xi-\xi_{0}\right) \mathbf{n}_{P F}(\mathbf{L}) \text { with } \xi_{0} \in \mathbb{R} .
$$

Proof. Let $\mathbf{p} \in \mathscr{P}_{c}$ and

$$
\mathbf{q}=\mathbf{P} \mathbf{p} \in \mathscr{C}^{2}\left(\mathbb{R}, \mathbb{C}^{N}\right) \cap \mathscr{L}^{\infty}\left(\mathbb{R}, \mathbb{C}^{N}\right) .
$$

Multiplying $(T W[c])$ on the left by $\mathbf{P}$, we get

$$
-\mathbf{q}^{\prime \prime}-c \mathbf{q}^{\prime}=\mathbf{J} \mathbf{q}-b\left[\mathbf{P}^{-1} \mathbf{q}\right] \mathbf{q} \text { in } \mathbb{R},
$$

and in particular

$$
-q_{1}^{\prime \prime}-c q_{1}^{\prime}=\left(\lambda_{P F}(\mathbf{L})-b\left[\mathbf{P}^{-1} \mathbf{q}\right]\right) q_{1} \text { in } \mathbb{R} .
$$

Since

$$
\begin{aligned}
\left(\boldsymbol{\Pi}_{P F}(\mathbf{L}) \mathbf{p}\right)^{T} \mathbf{n}_{P F}(\mathbf{L}) & =\left(\mathbf{P}^{-1} \operatorname{diag}\left(\mathbf{e}_{1}\right) \mathbf{q}\right)^{T} \mathbf{n}_{P F}(\mathbf{L}) \\
& =q_{1}\left(\mathbf{P}^{-1} \mathbf{e}_{1}\right)^{T} \mathbf{n}_{P F}(\mathbf{L}) \\
& =q_{1},
\end{aligned}
$$

$q_{1}$ is real-valued and in fact positive in $\mathbb{R}$.

First, let us verify that $\frac{q_{k}}{q_{1}}$ is globally bounded in $\mathbb{R}$ for all $k \in[N] \backslash\{1\}$. It is bounded in $(-\infty, 0]$ since $\inf _{(-\infty, 0]} q_{1}>0$ by [16, Theorem 1.5, iii)]. It is bounded in $[0,+\infty)$ since a left-multiplication of the first equivalent of Theorem 1.1 by $\mathbf{P}$ yields

whence

$$
\mathbf{q}(\xi) \sim A \xi^{k} \mathrm{e}^{-\frac{1}{2}\left(c-\sqrt{c^{2}-4 \lambda_{P F}(\mathbf{L})}\right) \xi} \mathbf{e}_{1}
$$

$$
\limsup _{+\infty}\left|\frac{q_{k}}{q_{1}}\right|=0
$$

Next, let us show by induction that $q_{N+1-k}=0$ in $\mathbb{R}$ for all $k \in[N-1]$.

- Basis: $k=1$. Due to the special form of $\mathbf{J}$, the equation satisfied by $q_{N}$ is

$$
-q_{N}^{\prime \prime}-c q_{N}^{\prime}=\left(\lambda_{1}-b\left[\mathbf{P}^{-1} \mathbf{q}\right]\right) q_{N} \text { in } \mathbb{R} .
$$

Define $z=\frac{q_{N}}{q_{1}}$ and $w=|z|^{2}$. The function $w$ is nonnegative and globally bounded. From

$$
\begin{gathered}
z^{\prime}=\frac{q_{N}^{\prime}}{q_{1}}-\frac{q_{1}^{\prime}}{q_{1}} z, \\
z^{\prime \prime}=\frac{q_{N}^{\prime \prime}}{q_{1}}-\frac{q_{1}^{\prime \prime}}{q_{1}} z-\frac{2 q_{1}^{\prime}}{q_{1}} z^{\prime},
\end{gathered}
$$


it follows

$$
-z^{\prime \prime}-\frac{q_{1} c+2 q_{1}^{\prime}}{q_{1}} z^{\prime}-\frac{q_{1}^{\prime \prime}+c q_{1}^{\prime}}{q_{1}} z=\left(\lambda_{1}-b\left[\mathbf{P}^{-1} \mathbf{q}\right]\right) z \text { in } \mathbb{R} .
$$

Using the equality satisfied by $q_{1}$, this equation reads:

$$
-z^{\prime \prime}-\frac{q_{1} c+2 q_{1}^{\prime}}{q_{1}} z^{\prime}+\left(\lambda_{P F}(\mathbf{L})-\lambda_{1}\right) z=0 \text { in } \mathbb{R} .
$$

Now, multiplying by $\bar{z}$, taking the real part, defining

$$
\gamma=2\left(\lambda_{P F}(\mathbf{L})-\operatorname{Re}\left(\lambda_{1}\right)\right)>0
$$

and using the obvious equality

$$
\begin{aligned}
\operatorname{Re}\left(z^{\prime \prime} \bar{z}\right) & =\operatorname{Re}(z)^{\prime \prime} \operatorname{Re}(z)+\operatorname{Im}(z)^{\prime \prime} \operatorname{Im}(z) \\
& =\frac{1}{2} w^{\prime \prime}-\left(\operatorname{Re}(z)^{\prime}\right)^{2}-\left(\operatorname{Im}(z)^{\prime}\right)^{2},
\end{aligned}
$$

it follows

$$
-w^{\prime \prime}-\frac{q_{1} c+2 q_{1}^{\prime}}{q_{1}} w^{\prime}+\gamma w \leq 0 \text { in } \mathbb{R} .
$$

This inequality implies the nonexistence of local maxima of $w$. Since $w \in$ $\mathscr{C}^{1}(\mathbb{R})$, there exists consequently $\xi_{0} \in \overline{\mathbb{R}}$ such that $w$ is decreasing on $\left(-\infty, \xi_{0}\right)$ and increasing on $\left(\xi_{0},+\infty\right)$. Therefore $w$ has well-defined limits at $\pm \infty$ and since $w \in \mathscr{L}^{\infty}(\mathbb{R})$, these limits are finite. By classical elliptic regularity and the Harnack inequality (see Gilbarg-Trudinger [14]) applied to the equation satisfied by $q_{1}, \frac{q_{1}^{\prime}}{q_{1}}$ is bounded in $\mathbb{R}$. By elliptic regularity again, applied this time to the equation

$$
-w^{\prime \prime}-\frac{q_{1} c+2 q_{1}^{\prime}}{q_{1}} w^{\prime}+\gamma w=-2\left(\operatorname{Re}(z)^{\prime}\right)^{2}-2\left(\operatorname{Im}(z)^{\prime}\right)^{2},
$$

the limits of $w$ have to be null, whence $w$ itself is null, and then $q_{N}$ is null.

- Inductive step: let $k \in[N-1] \backslash\{1\}$ and assume $q_{N+1-k}=0$. Defining

$$
\lambda=j_{N-k, N-k} \in \operatorname{sp} \mathbf{L} \backslash\left\{\lambda_{P F}(\mathbf{L})\right\},
$$

the equation satisfied by $q_{N+1-(k+1)}=q_{N-k}$ is

$$
-q_{N-k}^{\prime \prime}-c q_{N-k}^{\prime}=\left(\lambda-b\left[\mathbf{P}^{-1} \mathbf{q}\right]\right) q_{N-k} \text { in } \mathbb{R} .
$$

Repeating the argument detailed in the previous step shows similarly that $q_{N-k}$ is null.

Hence the proof by induction is ended and yields indeed $\mathbf{q}=q_{1} \mathbf{e}_{1}$ in $\mathbb{R}$. Now, back to the equation satisfied by $q_{1}$, we find

$$
-q_{1}^{\prime \prime}-c q_{1}^{\prime}=\left(\lambda_{P F}(\mathbf{L})-b\left[q_{1} \mathbf{n}_{P F}(\mathbf{L})\right]\right) q_{1} \text { in } \mathbb{R},
$$

which implies in view of well-known results on the traveling wave equation for the scalar KPP equation the existence of $\xi_{0} \in \mathbb{R}$ such that $q_{1}$ coincides with $\xi \mapsto$ $p_{c}\left(\xi-\xi_{0}\right)$. 
3.3. Global asymptotic stability. The auxiliary functions used in the proof of Proposition 3.1 can be used again to prove the global asymptotic stability of $\mathbf{v}^{\star}$ as stated in Theorem 1.3. In particular, the following lemma will be used repeatedly.

Lemma 3.2. There exists $\gamma>0$ such that all bounded positive classical solutions $\mathbf{u}$ of $\left(E_{K P P}\right)$ set in $(0,+\infty) \times \mathbb{R}$ satisfying

$$
\inf _{(t, x) \in(0,+\infty) \times \mathbb{R}} \mathbf{n}_{P F}(\mathbf{L})^{T} \boldsymbol{\Pi}_{P F}(\mathbf{L}) \mathbf{u}(t, x)>0
$$

satisfy also

$$
\lim _{t \rightarrow+\infty}\left(e^{\gamma t} \sup _{x \in \mathbb{R}}\left|\left(\mathbf{I}-\mathbf{\Pi}_{P F}(\mathbf{L})\right) \mathbf{u}(t, x)\right|\right)=0 .
$$

Proof. The proof is very similar to the first part of that of Proposition 3.1. Defining $\mathbf{v}=\mathbf{P u}$, the equation satisfied by $v_{1}$ is

$$
\partial_{t} v_{1}-\partial_{x x} v_{1}=\left(\lambda_{P F}(\mathbf{L})-b\left[\mathbf{P}^{-1} \mathbf{v}\right]\right) v_{1} \text { in }(0,+\infty) \times \mathbb{R} .
$$

For all $k \in[N] \backslash\{1\}$, there exists $\gamma_{k}>0$ such that $v_{k}$ satisfies

$$
\left\{\begin{array}{c}
\partial_{t}\left(\left|\frac{v_{k}}{v_{1}}\right|^{2}\right)-\partial_{x x}\left(\left|\frac{v_{k}}{v_{1}}\right|^{2}\right)-\frac{2 \partial_{x} v_{1}}{v_{1}} \partial_{x}\left(\left|\frac{v_{k}}{v_{1}}\right|^{2}\right)+\gamma_{k}\left|\frac{v_{k}}{v_{1}}\right|^{2} \leq 0 \quad \text { in }(0,+\infty) \times \mathbb{R} \\
\left(\left|\frac{v_{k}}{v_{1}}\right|^{2}\right)_{\mid\{0\} \times \mathbb{R}} \in \mathscr{L}^{\infty}(\mathbb{R},[0,+\infty)),
\end{array}\right.
$$

that is such that $z_{k}:(t, x) \mapsto \mathrm{e}^{\frac{\gamma_{k}}{2} t}\left|\frac{v_{k}}{v_{1}}\right|^{2}$ satisfies

$$
\left\{\begin{array}{c}
\partial_{t} z_{k}-\partial_{x x} z_{k}-\frac{2 \partial_{x} v_{1}}{v_{1}} \partial_{x} z_{k}+\frac{\gamma_{k}}{2} z_{k} \leq 0 \quad \text { in }(0,+\infty) \times \mathbb{R} \\
\left(z_{k}\right)_{\mid\{0\} \times \mathbb{R}} \in \mathscr{L}^{\infty}(\mathbb{R},[0,+\infty)) .
\end{array}\right.
$$

Since $z_{k}$ stays bounded locally in time, by a classical argument (detailed for instance in [16, Proposition 3.4]), $z_{k}$ vanishes uniformly in space as $t \rightarrow+\infty$. Consequently,

$$
\mathrm{e}^{\frac{\gamma_{k}}{4} t} \sup _{x \in \mathbb{R}}\left|v_{k}\right| \rightarrow 0 \text { as } t \rightarrow+\infty
$$

The conclusion follows from $\gamma=\min _{k \in[N]} \frac{\gamma_{k}}{4}$ and the following obvious algebraic equality:

$$
\left(\mathbf{I}-\boldsymbol{\Pi}_{P F}(\mathbf{L})\right) \mathbf{u}=\mathbf{P}^{-1}\left(\sum_{k=2}^{N} v_{k} \mathbf{e}_{k}\right) .
$$

We begin with the case of homogeneous initial data, which does not require $\mathbf{d}=\mathbf{1}_{N, 1}$ since $\left(E_{K P P}\right)$ reduces to $\left(E_{K P P}^{0}\right)$ in this context.

Proposition 3.3. All positive classical solutions of $\left(E_{K P P}^{0}\right)$ set in $(0,+\infty)$ converge as $t \rightarrow+\infty$ to $\mathbf{v}^{\star}$.

Proof. Once again, the proof is very similar to that of Proposition 3.1 .

Fix a positive classical solution $\mathbf{v}$ of $\left(E_{K P P}^{0}\right)$. By [16, Theorem 1.1], $\mathbf{v}(1) \gg \mathbf{0}$. Hence the function $\mathbf{u}: t \mapsto \mathbf{v}(t+1)$ is a classical solution of $\left(E_{K P P}^{0}\right)$ set in $(0,+\infty)$ which is positive in $[0,+\infty)$ (whereas $\mathbf{v}(0)$ might have null components) and which converges to $\mathbf{v}^{\star}$ if and only if $\mathbf{v}$ converges to $\mathbf{v}^{\star}$. 
The function $u=\mathbf{n}_{P F}(\mathbf{L})^{T} \boldsymbol{\Pi}_{P F}(\mathbf{L}) \mathbf{u}$ satisfies

$$
u^{\prime}=\lambda_{P F}(\mathbf{L}) u-b[\mathbf{u}] u .
$$

In order to apply Lemma 3.2 , it suffices to verify

$$
\inf _{t \in(0,+\infty)} u(t)>0
$$

On one hand, since $\mathbf{u}$ is positive in $[0,+\infty), u$ is positive in $[0,+\infty)$ as well. Hence any $t>0$ such that $u^{\prime}(t)=0$ is such that $b(\mathbf{u}(t))=\lambda_{P F}(\mathbf{L})$ and consequently any local minimum is larger than some positive constant. On the other hand, $\liminf _{t \rightarrow+\infty} u>0$ is a direct consequence of the persistence result [16, Theorem 1.3].

Since $b$ is Lipschitz-continuous on the compact set $\{\mathbf{v} \in \mathbf{K} \mid \mathbf{v} \leq \mathbf{k}\}$, there exists $C_{1}>0$ such that

$$
\left|b\left[u \mathbf{n}_{P F}(\mathbf{L})\right]-b[\mathbf{u}]\right| \leq C_{1}\left|\left(\mathbf{I}-\Pi_{P F}(\mathbf{L})\right) \mathbf{u}\right| \text { in }[0,+\infty),
$$

Now $u$ satisfies

$$
u^{\prime}=\lambda_{P F}(\mathbf{L}) u-b\left[u \mathbf{n}_{P F}(\mathbf{L})\right] u+\left(b\left[u \mathbf{n}_{P F}(\mathbf{L})\right]-b[\mathbf{u}]\right) u,
$$

with, by Lemma 3.2 .

$$
\left(b\left[u \mathbf{n}_{P F}(\mathbf{L})\right]-b[\mathbf{u}]\right) u=o(u) \text { as } t \rightarrow+\infty .
$$

It follows easily (see for instance 24]) that $u$ converges to the unique constant $\alpha^{\star}>0$ such that $\lambda_{P F}(\mathbf{L})=b\left[\alpha^{\star} \mathbf{n}_{P F}(\mathbf{L})\right]$, which precisely means

$$
\lim _{t \rightarrow+\infty} \mathbf{u}(t)=\mathbf{v}^{\star} \text {. }
$$

Finally, at the expense of assuming $\mathbf{d}=\mathbf{1}_{N, 1}$, we extend the previous result to non-homogeneous initial data.

Proposition 3.4. Assume $\mathbf{d}=\mathbf{1}_{N, 1}$. Then, for all bounded intervals $I \subset \mathbb{R}$, all bounded positive classical solutions $\mathbf{u}$ of $\left(E_{K P P}\right)$ set in $(0,+\infty) \times \mathbb{R}$ satisfy

$$
\lim _{t \rightarrow+\infty} \sup _{x \in I}\left|\mathbf{u}(t, x)-\mathbf{v}^{\star}\right|=0 .
$$

Consequently, if $\mathbf{d}=\mathbf{1}_{N, 1}$, the set of bounded nonnegative classical solutions of $\left(S_{K P P}\right)$ is exactly $\left\{\mathbf{0}, \mathbf{v}^{\star}\right\}$.

Proof. Let $\left(t_{n}\right)_{n \in \mathbb{N}} \in(0,+\infty)^{\mathbb{N}}$ such that $\lim _{n \rightarrow+\infty} t_{n}=+\infty$. Then, by classical parabolic estimates (Lieberman [27]) and a diagonal extraction process, the sequence

$$
\left(\mathbf{u}_{n}\right)_{n \in \mathbb{N}}=\left((t, x) \mapsto \mathbf{u}\left(t+t_{n}, x\right)\right)_{n \in \mathbb{N}}
$$

converges up to extraction to an entire classical solution of $\left(E_{K P P}\right)$ valued in $\prod_{i=1}^{N}\left[\nu, g_{i}(0)\right]$ (see [16, Theorems 1.2 and 1.3]).

Now let us prove that $\mathbf{v}^{\star}$ is the unique bounded entire classical solution $\tilde{\mathbf{u}}$ of $\left(E_{K P P}\right)$ satisfying

$$
\left(\inf _{\mathbb{R}^{2}} \tilde{u}_{i}\right)_{i \in[N]} \gg \mathbf{0}
$$

Let $\tilde{\mathbf{u}}$ be such a solution. The function $\tilde{u}=\mathbf{n}_{P F}(\mathbf{L})^{T} \boldsymbol{\Pi}_{P F}(\mathbf{L}) \tilde{\mathbf{u}}$ satisfies

$$
\partial_{t} \tilde{u}-\partial_{x x} \tilde{u}=\lambda_{P F}(\mathbf{L}) \tilde{u}-b\left[\tilde{u} \mathbf{n}_{P F}(\mathbf{L})\right] \tilde{u}+\left(b\left[\tilde{u} \mathbf{n}_{P F}(\mathbf{L})\right]-b[\tilde{\mathbf{u}}]\right) \tilde{u} .
$$


For all $\tau \in \mathbb{R}$,

$$
\inf _{(t, x) \in(0,+\infty) \times \mathbb{R}} \tilde{u}(t+\tau, x)>0 .
$$

By Lemma 3.2 , there exists $C>0$ such that, for all $t>0$ and all $\tau \in \mathbb{R}$,

$$
\sup _{x \in \mathbb{R}}\left|\tilde{u}(t+\tau, x) \mathbf{n}_{P F}(\mathbf{L})-\tilde{\mathbf{u}}(t+\tau, x)\right| \leq C \mathrm{e}^{-\gamma t} .
$$

It follows that for all $t>0$,

$$
\sup _{\left(t^{\prime}, x\right) \in \mathbb{R}^{2}}\left|\tilde{u}\left(t^{\prime}, x\right) \mathbf{n}_{P F}(\mathbf{L})-\tilde{\mathbf{u}}\left(t^{\prime}, x\right)\right| \leq C \mathrm{e}^{-\gamma t}
$$

and then passing the right-hand side to the limit $t \rightarrow+\infty$, we find

$$
\tilde{u}\left(t^{\prime}, x\right) \mathbf{n}_{P F}(\mathbf{L})=\tilde{\mathbf{u}}\left(t^{\prime}, x\right) \text { for all }\left(t^{\prime}, x\right) \in \mathbb{R}^{2} .
$$

Consequently, $\tilde{u}$ satisfies

$$
\partial_{t} \tilde{u}-\partial_{x x} \tilde{u}=\lambda_{P F}(\mathbf{L}) \tilde{u}-b\left[\tilde{u} \mathbf{n}_{P F}(\mathbf{L})\right] \tilde{u} .
$$

By standard results on the scalar KPP equation, $\tilde{u}=\alpha^{\star}$ in $\mathbb{R}^{2}$, that is $\tilde{\mathbf{u}}=\mathbf{v}^{\star}$.

A standard compactness argument ends the proof.

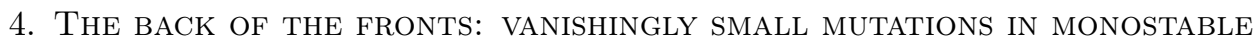

\section{TWO-COMPONENT SYSTEMS}

In this section, we assume $\left(H_{7}\right)$ and recall the existence and uniqueness of $(\mathbf{r}, \eta, \mathbf{m}) \in \mathrm{K}^{++} \times(0,+\infty) \times \mathrm{S}^{++}(\mathbf{0}, 1)$ such that

$$
\mathbf{L}=\mathbf{R}+\eta\left(\begin{array}{cc}
-1 & 1 \\
1 & -1
\end{array}\right) \mathbf{M} \text { with }(\mathbf{R}, \mathbf{M})=(\operatorname{diag}(\mathbf{r}), \operatorname{diag}(\mathbf{m})) .
$$

The various objects and notations of the problem now depend a priori on $\eta$ and a subscript $\eta_{\eta}$ might be added accordingly. The following definitions are recalled:

$$
\begin{gathered}
\alpha_{i}=\frac{r_{i}}{c_{i, i}} \text { for all } i \in\{1,2\}, \\
\mathbf{v}_{m}=\frac{1}{\operatorname{det} \mathbf{C}}\left(\begin{array}{l}
r_{1} c_{2,2}-r_{2} c_{1,2} \\
r_{2} c_{1,1}-r_{1} c_{2,1}
\end{array}\right) \text { if } \operatorname{det} \mathbf{C} \neq 0, \\
\mathscr{E}=\left\{(\eta, \mathbf{p}, c) \in(0,+\infty)^{2} \times \mathscr{C}^{2}\left(\mathbb{R}, \mathbb{R}^{2}\right) \mid \mathbf{p} \in \mathscr{P}_{c, \eta}, c \geq c_{\eta}^{\star}\right\}, \\
\partial_{t} \mathbf{u}-\mathbf{D} \partial_{x x} \mathbf{u}=\mathbf{R u}-(\mathbf{C u}) \circ \mathbf{u} . \quad\left(E_{K P P}\right)_{0}
\end{gathered}
$$

4.1. Preparatory lemmas. The proof of Theorem 1.5 will use the following lemmas which are of independent interest.

Lemma 4.1. Let $i \in\{1,2\}, j=3-i$ and

$$
\eta \in\left(0, \frac{r_{i} c_{i, j}}{m_{j} c_{i, i}}\right] .
$$

Then for all traveling wave solutions $(\mathbf{p}, c)$ of $\left(E_{K P P}\right)_{\eta}$,

$$
p_{i} \leq \alpha_{i} \text { in } \mathbb{R} \text {. }
$$

Remark. This lemma is straightforwardly generalizable to the case $N>2$. 
Proof. Having in mind the proof of [16, Theorem 1.5, ii)], it suffices to investigate the sign of

$r_{i} p_{i}-\eta m_{i} p_{i}+\eta m_{j} p_{j}-\left(c_{i, i} p_{i}+c_{i, j} p_{j}\right) p_{i}=p_{i}\left(r_{i}-\eta m_{i}-c_{i, i} p_{i}\right)+p_{j}\left(\eta m_{j}-c_{i, j} p_{i}\right)$.

This quantity is nonpositive provided

$$
p_{i} \geq \max \left(\frac{r_{i}-\eta m_{i}}{c_{i, i}}, \frac{\eta m_{j}}{c_{i, j}}\right) .
$$

Since

$$
\begin{gathered}
\frac{r_{i}}{c_{i, i}} \geq \frac{r_{i}-\eta m_{i}}{c_{i, i}} \text { for all } \eta \geq 0, \\
\frac{r_{i}}{c_{i, i}} \geq \frac{\eta m_{j}}{c_{i, j}} \text { for all } \eta \leq \frac{r_{i} c_{i, j}}{m_{j} c_{i, i}},
\end{gathered}
$$

we deduce indeed $p_{i} \leq \frac{r_{i}}{c_{i, i}}$.

Lemma 4.2. Let $i \in\{1,2\}, j=3-i$ and assume

$$
\frac{r_{i}}{r_{j}}>\frac{c_{i, j}}{c_{j, j}}
$$

Let

$$
\begin{gathered}
\bar{\eta}_{i}=\frac{1}{2} \min \left(\frac{r_{j} c_{j, i}}{m_{i} c_{j, j}}, \frac{r_{j}}{m_{i}}\left(\frac{r_{i}}{r_{j}}-\frac{c_{i, j}}{c_{j, j}}\right)\right), \\
\rho_{i}=\frac{1}{2} \frac{r_{j}}{c_{i, i}}\left(\frac{r_{i}}{r_{j}}-\frac{c_{i, j}}{c_{j, j}}\right) .
\end{gathered}
$$

Then for all $\rho \in\left(0, \rho_{i}\right]$, all $\eta \in\left(0, \bar{\eta}_{i}\right)$ and all traveling wave solutions $(\mathbf{p}, c)$ of $\left(E_{K P P}\right)_{\eta}$, there exists a unique

$$
\xi_{\rho} \in p_{i}^{-1}(\{\rho\}) .
$$

Furthermore $p_{i}$ is decreasing in $\left(\xi_{\rho},+\infty\right)$ and $p_{i}-\rho$ is positive in $\left(-\infty, \xi_{\rho}\right)$.

Remark. The following proof is mostly due to Griette-Raoul [18, Proposition 5.1].

Proof. Let $\zeta \in \mathbb{R}$ such that $p_{i}(\zeta)$ is a local minimum of $p_{i}$. Then

$$
r_{i} p_{i}(\zeta)-\eta m_{i} p_{i}(\zeta)+\eta m_{j} p_{j}(\zeta)-\left(c_{i, i} p_{i}(\zeta)+c_{i, j} p_{j}(\zeta)\right) p_{i}(\zeta) \leq 0 .
$$

This implies

$$
r_{i} p_{i}(\zeta)-\eta m_{i} p_{i}(\zeta)-\left(c_{i, i} p_{i}(\zeta)+c_{i, j} p_{j}(\zeta)\right) p_{i}(\zeta)<0,
$$

whence

$$
r_{i}-\eta m_{i}<c_{i, i} p_{i}(\zeta)+c_{i, j} p_{j}(\zeta),
$$

whence by Lemma 4.1

$$
r_{i}-\eta m_{i}<c_{i, i} p_{i}(\zeta)+c_{i, j} \frac{r_{j}}{c_{j, j}},
$$


and then

$$
\begin{aligned}
p_{i}(\zeta) & >\frac{1}{c_{i, i}}\left(r_{i}-\frac{r_{j} c_{i, j}}{c_{j, j}}\right)-\frac{\eta m_{i}}{c_{i, i}} \\
& >\frac{r_{j}}{c_{i, i}}\left(\frac{r_{i}}{r_{j}}-\frac{c_{i, j}}{c_{j, j}}\right)-\frac{\bar{\eta}_{i} m_{i}}{c_{i, i}} \\
& \geq \frac{1}{2} \frac{r_{j}}{c_{i, i}}\left(\frac{r_{i}}{r_{j}}-\frac{c_{i, j}}{c_{j, j}}\right) \\
& =\rho_{i} .
\end{aligned}
$$

Now let $\rho \in\left(0, \rho_{i}\right]$ and $\xi_{\rho} \in p_{i}^{-1}(\{\rho\})$.

Since $p_{i}\left(\xi_{\rho}\right)$ cannot be a local minimum, there exists a neighborhood of $\xi_{\rho}$ in which $p_{i}$ is strictly monotonic. Assume it is increasing. Then by continuity of $p_{i}^{\prime}$ and the previous estimate on local minima, $p_{i}$ is increasing in $\left(-\infty, \xi_{\rho}\right)$. By classical elliptic regularity, $\mathbf{p}$ converges as $\xi \rightarrow-\infty$ to a solution of $\mathbf{L v}=\mathbf{C v} \circ \mathbf{v}$, and by [16, Theorem 1.5, iii)], this solution is positive. But in view of the preceding estimates, necessarily

$$
\lim _{\xi \rightarrow-\infty} p_{i}(\xi)>\rho_{i} \geq p_{i}\left(\xi_{\rho}\right),
$$

which contradicts the monotonicity of $p_{i}$ in $\left(-\infty, \xi_{\rho}\right)$. Hence $p_{i}$ is decreasing in a neighborhood of $\xi_{\rho}$ and then in $\left(\xi_{\rho},+\infty\right)$. Consequently,

$$
p_{i}^{-1}(\{\rho\})=\left\{\xi_{\rho}\right\} .
$$

This holds for all $\rho \in\left(0, \rho_{i}\right]$ and therefore ends the proof.

4.2. Convergence at the back. Let $i \in\{1,2\}, j=3-i,\left(c_{\eta}\right)_{\eta \geq 0}$ and $\left(\mathbf{p}_{\eta}\right)_{\eta>0}$ such that

$$
\left\{\begin{array}{c}
\left(\eta, \mathbf{p}_{\eta}, c_{\eta}\right) \in \mathscr{E} \quad \text { for all } \eta>0, \\
c_{0}=\lim _{\eta \rightarrow 0} c_{\eta},
\end{array}\right.
$$

and assume from now on that

$$
\frac{r_{i}}{r_{j}}>\frac{c_{i, j}}{c_{j, j}}
$$

so that the assumptions of Theorem 1.5 are satisfied. Define subsequently

$$
\mathbf{v}_{s}=\left\{\begin{array}{cl}
\alpha_{i} \mathbf{e}_{i} & \text { if } \frac{r_{i}}{r_{j}} \geq \frac{c_{i, i}}{c_{j, i}} \\
\mathbf{v}_{m} & \text { if } \frac{r_{i}}{r_{j}}<\frac{c_{i, i}}{c_{j, i}} .
\end{array}\right.
$$

Proposition 4.3. There exists $\left(\zeta_{\eta}\right)_{\eta>0}$ such that, as $\eta \rightarrow 0,\left(\xi \mapsto \mathbf{p}_{\eta}\left(\xi+\zeta_{\eta}\right), c_{\eta}\right)_{\eta>0}$ converges up to extraction in $\mathscr{C}_{\text {loc }}^{2}\left(\mathbb{R}, \mathbb{R}^{2}\right) \times \mathbb{R}$ to a traveling wave solution $\left(\mathbf{p}_{\text {back }}, c_{0}\right)$ of $\left(E_{K P P}\right)_{0}$ achieving one of the following connections:

(1) $\mathbf{0}$ to $\mathbf{v}_{s}$

(2) $\alpha_{j} \mathbf{e}_{j}$ to $\mathbf{v}_{s}$,

(3) $\mathbf{0}$ to $\alpha_{i} \mathbf{e}_{i}$ with $\mathbf{p}$ semi-extinct.

Proof. Let $\rho=\min \left(\rho_{i}, v_{s, i}\right)$. By virtue of Lemma 4.2 , for all $\eta>0$, there exists a unique $\zeta_{\eta}$ such that:

- $p_{\eta, i}$ is decreasing in $\left(\zeta_{\eta},+\infty\right)$,

- $p_{\eta, i}\left(\zeta_{\eta}\right)=\rho$,

- $p_{\eta, i}-\rho$ is positive in $\left(-\infty, \zeta_{\eta}\right)$. 
By Lemma 4.1, classical elliptic estimates (Gilbarg-Trudinger [14]) and a diagonal extraction process, $\left(\xi \mapsto \mathbf{p}_{\eta}\left(\xi+\xi_{\eta}\right)\right)_{\eta>0}$ converges in $\mathscr{C}_{\text {loc }}^{2}$ up to extraction. Let $\mathbf{p}$ be its limit. We have directly $\mathbf{0} \leq \mathbf{p} \leq \boldsymbol{\alpha}$ in $\mathbb{R}$. In view of the normalization, we also have:

- $p_{i}$ is nonincreasing in $(0,+\infty)$,

- $p_{i}(0)=\rho$,

- $p_{i}-\rho$ is nonnegative in $(-\infty, 0)$.

Let $\left(\xi_{n}\right)_{n \in \mathbb{N}}$ such that $\xi_{n} \rightarrow-\infty$ as $n \rightarrow+\infty$. Defining

$$
\hat{\mathbf{p}}_{n}: \xi \mapsto \mathbf{p}\left(\xi+\xi_{n}\right) \text { for all } n \in \mathbb{N},
$$

by classical elliptic estimates and a diagonal extraction process again, $\left(\hat{\mathbf{p}}_{n}\right)_{n \in \mathbb{N}}$ converges up to extraction in $\mathscr{C}_{l o c}^{2}$ to a function $\hat{\mathbf{p}}$ satisfying

$$
-\mathbf{D} \hat{\mathbf{p}}^{\prime \prime}-c \hat{\mathbf{p}}^{\prime}=\mathbf{R} \hat{\mathbf{p}}-(\mathbf{C} \hat{\mathbf{p}}) \circ \hat{\mathbf{p}}
$$

and such that

$$
(\rho, 0) \leq\left(\hat{p}_{i}, \hat{p}_{j}\right) \leq\left(\alpha_{i}, \alpha_{j}\right) .
$$

In particular, $\hat{\mathbf{p}}$ is a stationary solution of

$$
\left\{\begin{array}{cc}
\partial_{t} \mathbf{u}-\partial_{x x} \mathbf{u}-c_{0} \partial_{x} \mathbf{u}=\mathbf{R u}-(\mathbf{C u}) \circ \mathbf{u} & \text { in }(0,+\infty) \times \mathbb{R} \\
\mathbf{u}(0, x)=\hat{\mathbf{p}}(x) & \text { for all } x \in \mathbb{R} .
\end{array}\right.
$$

Applying the comparison principle for two-components competitive parabolic systems to $\hat{\mathbf{p}}$ and to the solution of

$$
\left\{\begin{array}{cc}
\partial_{t} \mathbf{u}-\partial_{x x} \mathbf{u}-c_{0} \partial_{x} \mathbf{u}=\mathbf{R} \mathbf{u}-(\mathbf{C u}) \circ \mathbf{u} & \text { in }(0,+\infty) \times \mathbb{R} \\
\left(u_{i}, u_{j}\right)(0, x)=\left(\rho, \sup \hat{p}_{j}\right) & \text { for all } x \in \mathbb{R},
\end{array}\right.
$$

which is homogeneous in space and is therefore the solution of

$$
\left\{\begin{array}{c}
\partial_{t} \mathbf{u}=\mathbf{R u}-(\mathbf{C u}) \circ \mathbf{u} \quad \text { in }(0,+\infty) \times \mathbb{R} \\
\left(u_{i}, u_{j}\right)(0, x)=\left(\rho, \sup \hat{p}_{j}\right) \quad \text { for all } x \in \mathbb{R},
\end{array}\right.
$$

we directly obtain $\hat{\mathbf{p}}=\mathbf{v}_{s}$ if $\sup \hat{p}_{j}>0$ and $\hat{\mathbf{p}}=\alpha_{i} \mathbf{e}_{i}$ if $\sup \hat{p}_{j}=0$. In other words, if $\mathbf{v}_{s}=\alpha_{i} \mathbf{e}_{i}, \hat{\mathbf{p}}=\alpha_{i} \mathbf{e}_{i}$, and if $\mathbf{v}_{s}=\mathbf{v}_{m}, \hat{\mathbf{p}} \in\left\{\mathbf{v}_{s}, \alpha_{i} \mathbf{e}_{i}\right\}$. Since $\mathbf{v}_{s}$ and $\alpha_{i} \mathbf{e}_{i}$ are isolated steady states and $\mathbf{p}$ is continuous, the last diagonal extraction was not necessary and $\left(\hat{\mathbf{p}}_{n}\right)_{n \in \mathbb{N}}$ converges indeed to $\hat{\mathbf{p}}$, that is

$$
\lim _{-\infty} \mathbf{p} \in\left\{\mathbf{v}_{s}, \alpha_{i} \mathbf{e}_{i}\right\} .
$$

Since $p_{i}$ is nonincreasing in $(0,+\infty)$, it converges as $\xi \rightarrow+\infty$. By classical elliptic regularity,

$$
\lim _{+\infty}\left(-d_{i} p_{i}^{\prime \prime}-c_{0} p_{i}^{\prime}\right)=0
$$

whence either

$$
\lim _{+\infty} p_{i}=0
$$

or $p_{j}$ converges as well, its limit being

$$
\lim _{+\infty} p_{j}=\frac{1}{c_{i, j}}\left(r_{i}-c_{i, i} \lim _{+\infty} p_{i}\right) .
$$

In the second case, using $-d_{j} p_{j}^{\prime \prime}-c_{0} p_{j}^{\prime} \rightarrow 0, p_{i}(0)=\rho$ and the monotonicity of $p_{i}$ in $(0,+\infty)$, we find $\lim _{+\infty} \mathbf{p} \in\left\{\alpha_{j} \mathbf{e}_{j}, \mathbf{0}\right\}$, which contradicts directly $\lim _{+\infty} p_{i}>0$. Hence $p_{i}$ converges to 0 . 
Subsequently, since $p_{j}$ is positive, every local minimum of $p_{j}$ satisfies

$$
r_{j} \leq c_{j, j} p_{j}(\xi)+c_{j, i} p_{i}(\xi),
$$

which proves that for all sequences $\left(\xi_{n}\right)_{n \in \mathbb{N}}$ such that $\xi_{n} \rightarrow+\infty$ and $p_{j}\left(\xi_{n}\right)$ is a local minimum of $p_{j}, p_{j}\left(\xi_{n}\right)$ converges to $\alpha_{j}$. But then, by $\mathscr{C}^{1}$ regularity, either $p_{j}$ is monotonic in a neighborhood of $+\infty$ or there exists a sequence $\left(\xi_{n}\right)_{n \in \mathbb{N}}$ such that $\xi_{n} \rightarrow+\infty, p_{j}\left(\xi_{n}\right)$ is a local minimum of $p_{j}$ and $\left(p_{j}\left(\xi_{n}\right)\right)_{n \in \mathbb{N}}$ converges to $\liminf _{+\infty} p_{j}$. It turns out that in both cases $p_{j}$ converges, the possible limits being 0 and $\alpha_{j}$.

Therefore $\mathbf{p}$ is a traveling wave achieving exactly one of the following connections:

(1) $\mathbf{0}$ to $\mathbf{v}_{s}$

(2) $\alpha_{j} \mathbf{e}_{j}$ to $\mathbf{v}_{s}$,

(3) $\mathbf{0}$ to $\alpha_{i} \mathbf{e}_{i}$ with $\alpha_{i} \mathbf{e}_{i} \neq \mathbf{v}_{s}$,

(4) $\alpha_{j} \mathbf{e}_{j}$ to $\alpha_{i} \mathbf{e}_{i}$ with $\alpha_{i} \mathbf{e}_{i} \neq \mathbf{v}_{s}$.

It remains to show that the third case is semi-extinct and the fourth case is impossible. We will actually prove both statements simultaneously by proving that $\lim _{-\infty} \mathbf{p}=\alpha_{i} \mathbf{e}_{i} \neq \mathbf{v}_{s}$ implies $p_{j}=0$ in $\mathbb{R}$.

Assume $\lim _{-\infty} \mathbf{p}=\alpha_{i} \mathbf{e}_{i}$ and $\mathbf{v}_{s}=\mathbf{v}_{m}$. Assume also by contradiction that $p_{j}$ is positive in $\mathbb{R}$.

Multiplying the equation

$$
-d_{j} p_{j}^{\prime \prime}-c_{0} p_{j}^{\prime}=\left(r_{j}-c_{j, j} p_{j}-c_{j, i} p_{i}\right) p_{j}
$$

by the function

we find

$$
\varphi: \xi \mapsto \mathrm{e}^{\frac{c_{0}}{d_{j}} \xi}
$$

$$
-d_{j}\left(\varphi p_{j}^{\prime}\right)^{\prime}=\left(r_{j}-c_{j, j} p_{j}-c_{j, i} p_{i}\right) \varphi p_{j} .
$$

Recall that $\mathbf{v}_{s}=\mathbf{v}_{m}$ implies $\frac{r_{i}}{r_{j}}<\frac{c_{i, i}}{c_{j, i}}$, that is $r_{j}-c_{j, i} \alpha_{i}>0$. Therefore the quantity

$$
\bar{\xi}=\sup \left\{\xi \in \mathbb{R} \mid \forall \zeta \in(-\infty, \xi) \quad r_{j}-c_{j, j} p_{j}(\zeta)-c_{j, i} p_{i}(\zeta)>0\right\}
$$

is well-defined in $\mathbb{R} \cup\{+\infty\}$. In $(-\infty, \bar{\xi}), \varphi p_{j}^{\prime}$ is decreasing. Since on one hand $\lim _{-\infty} \varphi=0$ and on the other hand $\lim _{-\infty} p_{j}^{\prime}=0$ by classical elliptic regularity, the limit of $\varphi p_{j}^{\prime}$ itself is 0 . Consequently, $\varphi p_{j}^{\prime}$ is negative in $(-\infty, \bar{\xi})$. It follows that $p_{j}$ itself is decreasing in $(-\infty, \bar{\xi})$. But then $\lim _{-\infty} p_{j}=0$ implies that $p_{j}$ is negative in $(-\infty, \bar{\xi})$, which obviously contradicts the positivity of $p_{j}$. This ends the proof.

\section{Discussion}

5.1. Why is it likely hopeless to search for a general result on the behavior at the back of the front? First of all, the linearization of $\left(S_{K P P}\right)$ at $\mathbf{0}$ being cooperative, it is natural to wonder whether the dynamics of $\left(E_{K P P}\right)$ near some constant positive solution $\mathbf{u}$ of $\left(S_{K P P}\right)$ might be purely competitive or cooperative. In general, neither is the case. The linearized reaction term at any constant solution $\mathbf{u}$ of $\left(S_{K P P}\right)$ is

$$
\mathbf{L}_{\mathbf{u}}=\mathbf{L}-\operatorname{diag}(\mathbf{c}(\mathbf{u}))-\left(\mathbf{u} \mathbf{1}_{1, N}\right) \circ D \mathbf{c}(\mathbf{u}) .
$$

In the Lotka-Volterra case where there exists $\mathbf{C} \gg \mathbf{0}$ such that $\mathbf{c}(\mathbf{v})=\mathbf{C v}$, it reads

$$
\mathbf{L}_{\mathbf{u}}=\mathbf{L}-\operatorname{diag}(\mathbf{C u})-\left(\mathbf{u} \mathbf{1}_{1, N}\right) \circ \mathbf{C} .
$$


On one hand, it is clear that if there exists $(i, j) \in[N]^{2}$ such that $l_{i, j}=0$, then $l_{\mathbf{u}, i, j}<0$. On the other hand, assuming that there exists $i \in[N]$ such that $l_{i, i} \leq 0$, we find

$$
-l_{i, i} u_{i}+u_{i} c_{i, i} u_{i}>0 .
$$

Since $\mathbf{L u}-(\mathbf{C u}) \circ \mathbf{u}=\mathbf{0}$, it follows

$$
\sum_{j \in[N] \backslash\{i\}}\left(l_{i, j} u_{j}-u_{i} c_{i, j} u_{j}\right)>0,
$$

whence there exists $j \in[N] \backslash\{i\}$ such that $l_{i, j} u_{j}-u_{i} c_{i, j} u_{j}>0$, that is such that

$$
l_{\mathbf{u}, i, j}=l_{i, j}-u_{i} c_{i, j}>0 .
$$

Hence the competitive dynamics and the cooperative dynamics are indeed intertwined near $\mathbf{u}$.

Next, in view of the literature on non-cooperative KPP systems, it could be tempting to conjecture the uniqueness and the local stability of the constant positive solution of $\left(S_{K P P}\right)$ (see for instance Dockery-Hutson-Mischaikow-Pernarowski [7] or Morris-Börger-Crooks [29]). However, if $\mathbf{c}$ is linear as before and if

$$
(N, \mathbf{L}, \mathbf{C})=\left(2, \mathbf{I}_{2}+\frac{1}{5}\left(\begin{array}{cc}
-1 & 1 \\
1 & -1
\end{array}\right), \frac{1}{10}\left(\begin{array}{ll}
1 & 9 \\
9 & 1
\end{array}\right)\right)
$$

then this property fails. Indeed, straightforward computations show that the set of constant positive solutions of $\left(S_{K P P}\right)$ is

$$
\left\{\left(\begin{array}{l}
3-\sqrt{\frac{15}{2}} \\
3+\sqrt{\frac{15}{2}}
\end{array}\right), \mathbf{1}_{2,1},\left(\begin{array}{l}
3+\sqrt{\frac{15}{2}} \\
3-\sqrt{\frac{15}{2}}
\end{array}\right)\right\} .
$$

From the associated linearizations, it is easily found that, with respect to $\left(E_{K P P}^{0}\right)$, the symmetric solution $\mathbf{1}_{2,1}$ is a saddle point whereas the other two solutions are stable nodes.

Last, we also point out that if $\mathbf{d}=\mathbf{1}_{2,1}$ then the preceding counter-example admits a family of traveling waves connecting $\mathbf{0}$ to the saddle point $\mathbf{1}_{2,1}$. Indeed, looking for profiles $\mathbf{p}$ of the form $\xi \mapsto p(\xi) \mathbf{1}_{2,1},(T W[c])$ reduces to

$$
-p^{\prime \prime}-c p^{\prime}=p-p^{2}
$$

which, by virtue of well-known results on the scalar KPP equation, admits solutions connecting 0 to 1 if and only if $c \geq 2$. Hence we cannot hope to prove that all traveling waves connect $\mathbf{0}$ to a stable steady state.

5.2. What about the general separated competition case, with $\mathbf{d}$ and a possibly different from $\mathbf{1}_{N, 1}$ ? The general case might be more subtle than expected, even regarding the ODE system $\left(E_{K P P}^{0}\right)$ : although the linearization at $\mathbf{v}^{\star}$

$$
\mathbf{L}_{\mathbf{v}^{\star}}=\mathbf{L}-\lambda_{\mathbf{a}} \mathbf{A}-\mathbf{A v}^{\star}\left(\nabla b\left(\mathbf{v}^{\star}\right)^{T}\right),
$$

seems to be adequately described as a matrix of the form $-\mathbf{P}-\mathbf{Q}$ with $\mathbf{P}=\lambda_{\mathbf{a}} \mathbf{A}-\mathbf{L}$ a singular M-matrix and $\mathbf{Q}=\mathbf{A} \mathbf{v}^{\star}\left(\nabla b\left(\mathbf{v}^{\star}\right)^{T}\right)$ a positive rank-one matrix, a recent paper by Bierkens and Ran 22 highlights thanks to a counter-example that such matrices can have eigenvalues with positive real part (and there is in addition a counter-example with irreducible $-\mathbf{P}$, so that irreducibility is not a sufficient condition to ensure all eigenvalues are negative). Therefore it is unclear whether 
$\mathbf{v}^{\star}$ is always locally asymptotically stable with respect to $\left(E_{K P P}^{0}\right)$. Actually, the main purpose of the study of Bierkens and Ran is to establish several conditions sufficient to guarantee that all eigenvalues have a negative real part (conditions among which we find $N=2$ and, of course, $\mathbf{a}=\mathbf{1}_{N, 1}$ ).

In the case $N=2$, classical calculations show that the system $\left(E_{K P P}\right)$ is not subjected to Turing instabilities with respect to periodic perturbations. Therefore it might be fruitful to investigate more thoroughly the two-component system. Nevertheless, to this day we do not have any further result.

5.3. Where does Conjecture 1.4 come from? Let us bring forth some insight into the limiting problem. What are the spreading properties of $\left(E_{K P P}\right)_{0}$ with respect to front-like initial data? What are the propagating solutions of $\left(E_{K P P}\right)_{0}$ invading the null state?

Concerning the bistable case, we have at our disposal a recent result by Carrère [5] which can be summed up as follows. Consider the Cauchy problem where $(-\infty, 0)$ is initially inhabited mostly but not only (in a sense made rigorous by Carrère) by $u_{1}$ and $(0,+\infty)$ is completely uninhabited. Let $c_{\alpha_{1} \mathbf{e}_{1} \rightarrow \alpha_{2} \mathbf{e}_{2}}$ be the speed of the bistable front equal to $\alpha_{1} \mathbf{e}_{1}$ at $-\infty$ and to $\alpha_{2} \mathbf{e}_{2}$ at $+\infty$, as given by Kan-On [21] and Gardner [12]. Recall that the following bounds hold true:

$$
-2 \sqrt{d_{2} r_{2}}<c_{\alpha_{1} \mathbf{e}_{1} \rightarrow \alpha_{2} \mathbf{e}_{2}}<2 \sqrt{d_{1} r_{1}} .
$$

Carrère's theorem is then:

(1) if $2 \sqrt{d_{1} r_{1}}>2 \sqrt{d_{2} r_{2}}$ and $c_{\alpha_{1} \mathbf{e}_{1} \rightarrow \alpha_{2} \mathbf{e}_{2}}>0$, then asymptotically in time, $u_{2}$ is extinct and $u_{1}$ spreads at speed $2 \sqrt{d_{1} r_{1}}$;

(2) if $2 \sqrt{d_{1} r_{1}}<2 \sqrt{d_{2} r_{2}}$ and $c_{\alpha_{1} \mathbf{e}_{1} \rightarrow \alpha_{2} \mathbf{e}_{2}}>0$, then asymptotically in time, $u_{2}$ spreads on the right at speed $2 \sqrt{d_{2} r_{2}}$ but is then replaced by $u_{1}$ at speed $c_{\alpha_{1} \mathbf{e}_{1} \rightarrow \alpha_{2} \mathbf{e}_{2}}$

(3) if $2 \sqrt{d_{1} r_{1}}<2 \sqrt{d_{2} r_{2}}$ and $c_{\alpha_{1} \mathbf{e}_{1} \rightarrow \alpha_{2} \mathbf{e}_{2}}<0$, then asymptotically in time, $u_{2}$ chases $u_{1}$ on the left at speed $c_{\alpha_{1} \mathbf{e}_{1} \rightarrow \alpha_{2} \mathbf{e}_{2}}$ and spreads on the right at speed $2 \sqrt{d_{2} r_{2}}$.

This result was long-awaited but, as far as we know, Carrère's proof is the first one.

Up to the sign of $c_{\alpha_{1} \mathbf{e}_{1} \rightarrow \alpha_{2} \mathbf{e}_{2}}$, the second and the third cases above are identical. Recall that the sign of $c_{\alpha_{1} \mathbf{e}_{1} \rightarrow \alpha_{2} \mathbf{e}_{2}}$ is in general a tough problem, although recently some particular cases have been successfully solved (strong competition in Girardin-Nadin [17, special choices of parameter values in Guo-Lin [19], perturbation of the standing wave in Risler [31]).

A natural conjecture in view of Carrère's result is the long-time convergence, in the first case, to a traveling wave connecting $\mathbf{0}$ to $\alpha_{1} \mathbf{e}_{1}$ at speed $2 \sqrt{d_{1} r_{1}}$ and with a semi-extinct profile $\mathbf{p}=p \mathbf{e}_{1}$. However, in the second and third cases, a more complex limit seems to arise.

The entire solutions connecting three or more stationary states with decreasingly ordered speeds were first described in the scalar setting by Fife and McLeod [10] and are referred to as propagating terraces, or simply terraces, since the work of Ducrot, Giletti and Matano [8]. A terrace with $n-1$ intermediate states is defined as a finite family of traveling waves $\left(\left(\mathbf{p}_{i}, c_{i}\right)\right)_{i \in[n]}$ such that $\mathbf{p}_{i}(-\infty)=\mathbf{p}_{i+1}(+\infty)$ for all $i \in[n-1]$ and such that $\left(c_{i}\right)_{i \in[n]}$ is decreasing. Provided the uniqueness (up to translation of the profile) of the traveling wave connecting $\mathbf{v}_{i}=\mathbf{p}_{i}(+\infty)$ 
to $\mathbf{v}_{i+1}=\mathbf{p}_{i}(-\infty)$ at speed $c_{i}$, the terrace is equivalently defined as the family $\left(\left(\mathbf{v}_{i}, c_{i}\right)_{i \in[n]}, \mathbf{v}_{n+1}\right)$. However, in general, this family only defines a family of terraces that will be denoted hereafter $\mathscr{T}\left(\left(\mathbf{v}_{i}, c_{i}\right)_{i \in[n]}, \mathbf{v}_{n+1}\right)$.

In terms of this definition, the expected limits in the second and third cases studied by Carrère are terraces belonging to

$$
\mathscr{T}\left(\mathbf{0}, 2 \sqrt{d_{2} r_{2}}, \alpha_{2} \mathbf{e}_{2}, c_{\alpha_{1} \mathbf{e}_{1} \rightarrow \alpha_{2} \mathbf{e}_{2}}, \alpha_{1} \mathbf{e}_{1}\right)
$$

with a semi-extinct first profile.

The obvious conjecture is then that all propagating solutions invading $\mathbf{0}$ apart from semi-extinct monostable traveling waves belong to

$$
\bigcup_{i \in\{1,2\}} \bigcup_{c \geq 2 \sqrt{d_{i} r_{i}}} \mathscr{T}\left(\mathbf{0}, c, \alpha_{i} \mathbf{e}_{i}, c_{\alpha_{3-i} \mathbf{e}_{3-i} \rightarrow \alpha_{i} \mathbf{e}_{i}}, \alpha_{3-i} \mathbf{e}_{3-i}\right)
$$

and have a semi-extinct first profile.

The bistable case being more or less understood, we now turn our attention to the monostable case. Let $\mathbf{v}_{s} \in\left\{\alpha_{1} \mathbf{e}_{1}, \alpha_{2} \mathbf{e}_{2}, \mathbf{v}_{m}\right\}$ be the unique stable state, $\mathbf{v}_{u} \in\left\{\mathbf{0}, \alpha_{1} \mathbf{e}_{1}, \alpha_{2} \mathbf{e}_{2}\right\}$ be an unstable state and consider the Cauchy problem with compactly supported perturbations of $\mathbf{v}_{u}$ as initial data. Although the case $\mathbf{v}_{u}=$ $\alpha_{i} \mathbf{e}_{i}$ with

$$
i \in \mathrm{I}_{u}=\left\{j \in\{1,2\} \mid \alpha_{j} \mathbf{e}_{j} \neq \mathbf{v}_{s}\right\} .
$$

is well understood (Lewis, Li and Weinberger proved the uniqueness of the spreading speed $c_{\mathbf{v}_{s} \rightarrow \alpha_{i} \mathbf{e}_{i}}^{\star}$ [25, 34]), the case $\mathbf{v}_{u}=\mathbf{0}$ is much more intricate: in particular, for $\mathbf{v}_{s}=\mathbf{v}_{m}$, a recent theorem analogous to that of Carrère and due to Lin and Li [28] shows that if $d_{2} r_{2}>d_{1} r_{1}$, then $u_{2}$ will invade first at speed $2 \sqrt{d_{2} r_{2}}$ and then be chased by $u$. Although straightforward comparisons show that the replacement occurs somewhere in $\left[c_{\mathbf{v}_{m} \rightarrow \alpha_{2} \mathbf{e}_{2}}^{\star} t, 2 \sqrt{d_{1} r_{1}} t\right]$, the exact speed of $u$ is a delicate question, unsettled in the paper of Lin and Li.

Tang and Fife 32 established by phase-plane analysis that traveling waves connecting $\mathbf{0}$ to $\mathbf{v}_{s}$ exist if and only if the speed $c$ satisfies $c \geq c_{\mathbf{v}_{s} \rightarrow \mathbf{0}}^{T W}$, where

$$
c_{\mathbf{v}_{s} \rightarrow \mathbf{0}}^{T W}=2 \sqrt{\max _{i \in\{1,2\}} d_{i} r_{i}}
$$

is linearly determinate.

Terraces connecting $\mathbf{0}$ to $\mathbf{v}_{s}$ through an intermediate unstable state $\alpha_{i} \mathbf{e}_{i}$ with $i \in \mathrm{I}_{u}$ should involve semi-extinct monostable traveling waves connecting $\mathbf{0}$ to $\alpha_{i} \mathbf{e}_{i}$ and monostable traveling waves connecting $\alpha_{i} \mathbf{e}_{i}$ to $\mathbf{v}_{s}$. Again, there exists a minimal wave speed $c_{\mathbf{v}_{s} \rightarrow \alpha_{i} \mathbf{e}_{i}}^{T W}$, as proved for instance by Kan-On [22] or Lewis-Li-Weinberger [26]. Recall that $c_{\mathbf{v}_{s} \rightarrow \alpha_{i} \mathbf{e}_{i}}^{T W}$ is not linearly determinate in general, however it is bounded from below by the linear speed:

$$
c_{\mathbf{v}_{s} \rightarrow \alpha_{i} \mathbf{e}_{i}}^{T W} \geq 2 \sqrt{d_{3-i} r_{3-i}\left(1-\frac{c_{3-i, i} r_{i}}{c_{i, i} r_{3-i}}\right)} .
$$

In any case, it is natural to expect that for all $i \in \mathrm{I}_{u}$, terraces belonging to $\mathscr{T}\left(\mathbf{0}, c, \alpha_{i} \mathbf{e}_{i}, c^{\prime}, \mathbf{v}_{s}\right)$ with a semi-extinct first profile exist if and only if

$$
\left\{\begin{array}{c}
c_{\mathbf{v}_{s} \rightarrow \alpha_{i} \mathbf{e}_{i}} \leq c^{\prime} \\
2 \sqrt{d_{i} r_{i}} \leq c \\
c^{\prime}<c
\end{array}\right.
$$


Consequently, the conjecture is that all propagating solutions invading $\mathbf{0}$ apart from (possibly semi-extinct) monostable traveling waves belong to

$$
\bigcup_{i \in \mathbf{I}_{u}} \bigcup_{c \geq 2 \sqrt{d_{i} r_{i}}} \bigcup_{c^{\prime} \geq c_{\mathbf{v}_{s} \rightarrow \alpha_{i} \mathbf{e}_{i}}} \mathscr{T}\left(\mathbf{0}, c, \alpha_{i} \mathbf{e}_{i}, c^{\prime}, \mathbf{v}_{s}\right)
$$

and have a semi-extinct first profile.

Having these conjectures in mind, we introduce small mutations and wonder how they affect the outcome. An heuristic answer due to Elliott and Cornell [9] suggests that "the only role of mutations is to ensure that both morphs travel at the same speed". Therefore, there might exist functions $\mathbf{u}^{0}: \mathbb{R} \rightarrow \mathrm{K}$ such that the solutions $\left(\mathbf{u}_{\eta}\right)_{\eta \geq 0}$ of the Cauchy problem associated with $\left(E_{K P P}\right)_{\eta}$ with initial data $\mathbf{u}^{0}$ admit as long-time asymptotic a traveling wave if $\eta>0$ and a terrace of $\mathscr{T}\left(\mathbf{0}, c, \alpha_{i} \mathbf{e}_{i}, c^{\prime}, \mathbf{v}\right)$ if $\eta=0$. We refer hereafter to such traveling waves as quasi$\mathscr{T}\left(\mathbf{0}, c, \alpha_{i} \mathbf{e}_{i}, c^{\prime}, \mathbf{v}\right)$ traveling waves.

In order to study these special traveling waves, we resort to numerical simulations. We find two completely different behaviors.

- In the bistable case (Figure 5.1, quasi- $\mathscr{T}\left(\mathbf{0}, 2 \sqrt{d_{i} r_{i}}, \alpha_{i} \mathbf{e}_{i}, c_{\alpha_{j} \mathbf{e}_{j} \rightarrow \alpha_{i} \mathbf{e}_{i}}, \alpha_{j} \mathbf{e}_{j}\right)$ traveling waves (with $i \in\{1,2\}$ and $j=3-i$ ) converge as $\eta \rightarrow 0$ to a semiextinct traveling wave connecting $\mathbf{0}$ to $\alpha_{j} \mathbf{e}_{j}$ if $c_{\alpha_{j} \mathbf{e}_{j} \rightarrow \alpha_{i} \mathbf{e}_{i}}>0$ and to $\alpha_{i} \mathbf{e}_{i}$ if $c_{\alpha_{j} \mathbf{e}_{j} \rightarrow \alpha_{i} \mathbf{e}_{i}}<0$.

- In the monostable case (Figure 5.2), for all $i \in \mathrm{I}_{u}$, quasi- $\mathscr{T}\left(\mathbf{0}, 2 \sqrt{d_{i} r_{i}}, \alpha_{i} \mathbf{e}_{i}, c^{\prime}, \mathbf{v}_{s}\right)$ traveling waves connect $\mathbf{0}$ to $\mathbf{v}_{s}$ through an intermediate bump of $u_{i}$. As $\eta \rightarrow 0$, the amplitude of this bump tends to $\alpha_{i}$ while its length tends slowly to $+\infty$ (seemingly like $\ln \eta$ ). Therefore, depending on the normalization, the limit of the profiles as $\eta \rightarrow 0$ is either a semi-extinct connection between $\mathbf{0}$ and $\alpha_{i} \mathbf{e}_{i}$ or a monostable connection between $\alpha_{i} \mathbf{e}_{i}$ and $\mathbf{v}_{s}$.

5.4. Why is Conjecture 1.4 silent about the bistable case with $c_{\alpha_{1} \mathrm{e}_{1} \rightarrow \alpha_{2} \mathrm{e}_{2}}=$ 0 ? In this very special case, additional asymmetry assumptions on the coefficients are necessary in order to exclude connections between $\mathbf{0}$ and the saddle-point $\mathbf{v}_{m}$, as indicated by the following immediate proposition, built on a counter-example given in Subsection 5.1 .

Proposition 5.1. Assume $\left(H_{7}\right), \mathbf{d}=\mathbf{1}_{2,1}, \mathbf{r}=\mathbf{1}_{2,1}, \mathbf{m}=\frac{1}{\sqrt{2}} \mathbf{1}_{2,1}$ and the existence of $a \in(1,+\infty)$ such that

$$
\mathbf{C}=\left(\begin{array}{ll}
1 & a \\
a & 1
\end{array}\right)
$$

Then $\mathbf{v}_{m}=\frac{1}{\lambda_{P F}(\mathbf{C})} \mathbf{1}_{2,1} \in \mathrm{K}^{++}$is a saddle-point and, for all $\eta \geq 0$ and all $c \geq 2$, there exists a unique $p_{c, \eta} \in \mathscr{C}^{2}(\mathbb{R})$ such that

$$
\left\{\begin{array}{c}
p_{c, \eta} \mathbf{1}_{2,1} \in \mathscr{P}_{c, \eta} \\
p_{c, \eta}(0)=\frac{1}{2 \lambda_{P F}(\mathbf{C})} \\
\lim _{\xi \rightarrow-\infty} p_{c, \eta}(\xi)=\frac{1}{\lambda_{P F}(\mathbf{C})} .
\end{array}\right.
$$

In particular, $\left(p_{c, \eta} \mathbf{1}_{2,1}, c\right)$ connects $\mathbf{0}$ to $\mathbf{v}_{m}$.

Furthermore,

$$
(c, \eta) \mapsto p_{c, \eta} \in \mathscr{C}\left([2,+\infty) \times[0,+\infty), \mathscr{W}^{2, \infty}(\mathbb{R}, \mathbb{R})\right) .
$$




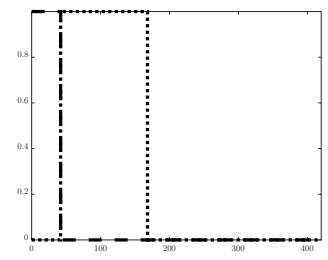

(A) $t=0$

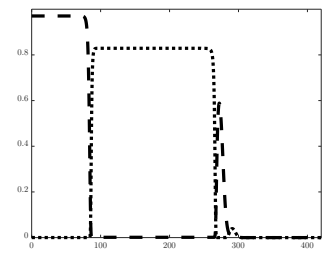

(D) $t=55$

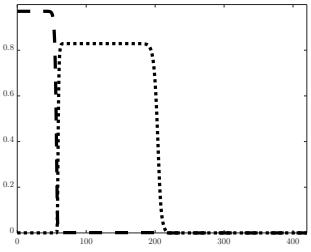

(в) $t=20$

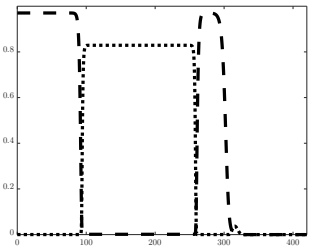

(Е) $t=65$

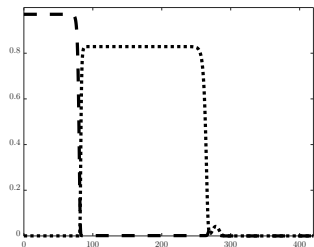

(c) $t=50$

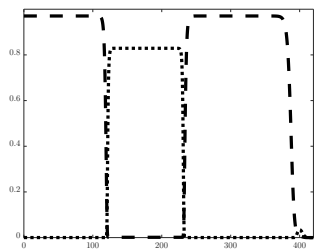

(F) $t=100$

Figure 5.1. Numerical simulation of the bistable case with initial data corresponding to a competition-diffusion terrace $\left(u_{1}\right.$ dashed line, $u_{2}$ dotted line, $x$ as horizontal axis).

Parameter values: $\mathbf{d}=(1,1.5125)^{T}, \mathbf{r}=\mathbf{1}_{2,1}, \mathbf{m}=\mathbf{1}_{2,1}, \eta=0.025$, $c_{1,1}=c_{2,2}=1, c_{1,2}=20, c_{2,1}=110$, so that [17] $c_{\alpha_{1} \mathbf{e}_{1} \rightarrow \alpha_{2} \mathbf{e}_{2}}>0$. The traveling wave which is on the right at $t=100$, driven by a very small bump of $u_{2}$ but dominated at the back by $u_{1}$, is the long-time asymptotic. Indeed the $u_{2}$-dominated area in the middle shrinks from both sides at a speed close to $\left|c_{\alpha_{1} \mathbf{e}_{1} \rightarrow \alpha_{2} \mathbf{e}_{2}}\right|$ and will ultimately disappear.

\section{ACKNOWLEDGMENTS}

The author thanks Grégoire Nadin for the attention he paid to this work and Elaine Crooks for fruitful discussions. He also thanks anonymous referees and the associate editor for very valuable comments which led to a much clearer manuscript.

\section{REFERENCES}

[1] Guy Barles, Lawrence C. Evans, and Panagiotis E. Souganidis. Wavefront propagation for reaction-diffusion systems of PDE. Duke Math. J., 61(3):835-858, 1990.

[2] Joris Bierkens and André Ran. A singular M-matrix perturbed by a nonnegative rank one matrix has positive principal minors; is it D-stable? Linear Algebra Appl., 457:191-208, 2014.

[3] Olivier Bonnefon, Jérome Coville, Jimmy Garnier, François Hamel, and Lionel Roques. The spatio-temporal dynamics of neutral genetic diversity. Ecological Complexity, 20:282-292, 2014.

[4] Jack Carr and Adam Chmaj. Uniqueness of travelling waves for nonlocal monostable equations. Proc. Amer. Math. Soc., 132(8):2433-2439, 2004.

[5] Cécile Carrère. Spreading speeds for a two-species competition-diffusion system. working paper or preprint, Mar 2017.

[6] Jérome Coville and Frédéric Fabre. Convergence to the equilibrium in a Lotka-Volterra ODE competition system with mutations. ArXiv e-prints, jan 2013. 


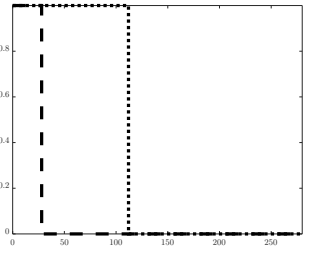

(A) $t=0$

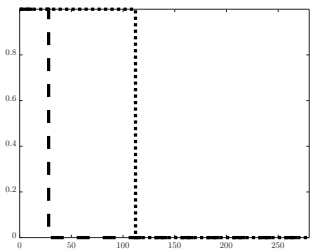

(D) $t=0$

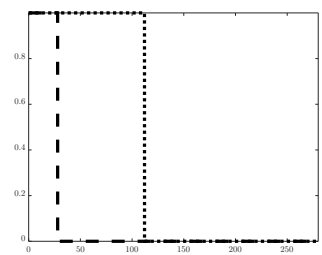

(G) $t=0$

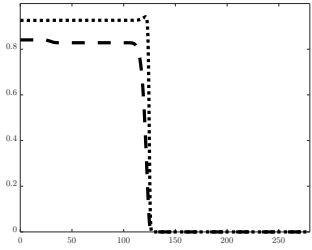

(в) $t=5$

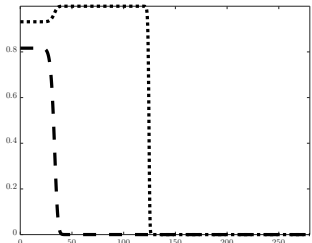

(Е) $t=5$

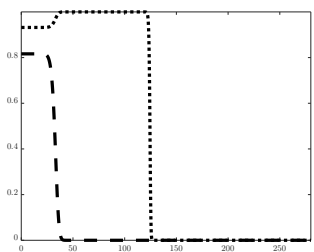

(н) $t=5$

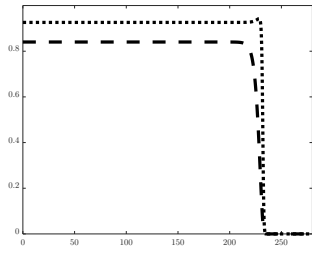

(c) $t=40$

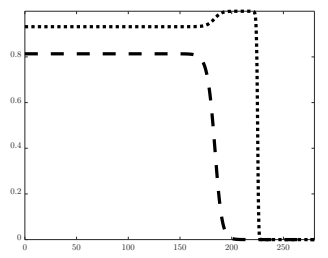

(F) $t=40$

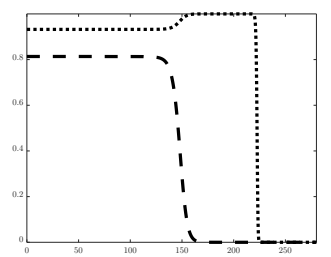

(I) $t=40$

Figure 5.2. Numerical simulations of the monostable case with initial data corresponding to a competition-diffusion terrace $\left(u_{1}\right.$ dashed line, $u_{2}$ dotted line, $x$ as horizontal axis).

Parameter values: $\mathbf{d}=\left(1, \frac{1}{3}\right)^{T}, \mathbf{r}=(1,6)^{T}, \mathbf{m}=\mathbf{1}_{2,1}, c_{1,1}=1$, $c_{2,2}=6, c_{1,2}=0.2, c_{2,1}=0.5, \eta=2.5 \times 10^{-1}$ on the first line, $\eta=2.5 \times 10^{-6}$ on the second line, $\eta=2.5 \times 10^{-11}$ on the third line.

[7] Jack Dockery, Vivian Hutson, Konstantin Mischaikow, and Mark Pernarowski. The evolution of slow dispersal rates: a reaction diffusion model. J. Math. Biol., 37(1):61-83, 1998.

[8] Arnaud Ducrot, Thomas Giletti, and Hiroshi Matano. Existence and convergence to a propagating terrace in one-dimensional reaction-diffusion equations. Trans. Amer. Math. Soc., 366(10):5541-5566, 2014.

[9] Elizabeth C. Elliott and Stephen J. Cornell. Dispersal polymorphism and the speed of biological invasions. PLOS ONE, 7(7):1-10, 072012.

[10] Paul C. Fife and J. B. McLeod. The approach of solutions of nonlinear diffusion equations to travelling front solutions. Archive for Rational Mechanics and Analysis, 65(4):335-361, 1977.

[11] Ronald Aylmer Fisher. The wave of advance of advantageous genes. Annals of eugenics, 7(4):355-369, 1937.

[12] Robert A. Gardner. Existence and stability of travelling wave solutions of competition models: a degree theoretic approach. J. Differential Equations, 44(3):343-364, 1982. 
[13] Jimmy Garnier, Thomas Giletti, François Hamel, and Lionel Roques. Inside dynamics of pulled and pushed fronts. Journal de Mathématiques Pures et Appliquées, 98(4):428-449, 2012.

[14] David Gilbarg and Neil S. Trudinger. Elliptic Partial Differential Equations of Second Order. Classics in Mathematics. Springer-Verlag, 2001.

[15] Michael E. Gilpin and Francisco J. Ayala. Global models of growth and competition. Proceedings of the National Academy of Sciences, 70(12):3590-3593, 1973.

[16] Léo Girardin. Non-cooperative Fisher-KPP systems: traveling waves and long-time behavior. ArXiv e-prints, dec 2016.

[17] Léo Girardin and Grégoire Nadin. Travelling waves for diffusive and strongly competitive systems: relative motility and invasion speed. European J. Appl. Math., 26(4):521-534, 2015.

[18] Quentin Griette and Gaël Raoul. Existence and qualitative properties of travelling waves for an epidemiological model with mutations. J. Differential Equations, 260(10):7115-7151, 2016.

[19] Jong-Shenq Guo and Ying-Chih Lin. The sign of the wave speed for the Lotka-Volterra competition-diffusion system. Commun. Pure Appl. Anal., 12(5):2083-2090, 2013.

[20] Jong-Shenq Guo and Chang-Hong Wu. Traveling wave front for a two-component lattice dynamical system arising in competition models. J. Differential Equations, 252(8):4357-4391, 2012.

[21] Yukio Kan-on. Parameter dependence of propagation speed of travelling waves for competition-diffusion equations. SIAM J. Math. Anal., 26(2):340-363, 1995.

[22] Yukio Kan-on. Fisher wave fronts for the lotka-volterra competition model with diffusion. Nonlinear Anal., 28(1):145-164, 1997.

[23] Andrei N. Kolmogorov, I. G. Petrovsky, and N. S. Piskunov. Étude de l'équation de la diffusion avec croissance de la quantité de matière et son application à un problème biologique. Bulletin Université d'État à Moscou, 1:1-25, 1937.

[24] Hélène Leman, Sylvie Méléard, and Sepideh Mirrahimi. Influence of a spatial structure on the long time behavior of a competitive lotka-volterra type system. Discrete Contin. Dyn. Syst. Ser. B, 20(2):469-493, 2015.

[25] Mark A. Lewis, Bingtuan Li, and Hans F. Weinberger. Spreading speed and linear determinacy for two-species competition models. J. Math. Biol., 45(3):219-233, 2002.

[26] Bingtuan Li, Hans F. Weinberger, and Mark A. Lewis. Spreading speeds as slowest wave speeds for cooperative systems. Math. Biosci., 196(1):82-98, 2005.

[27] Gary M. Lieberman. Second order parabolic differential equations. World Scientific Publishing Co., Inc., River Edge, NJ, 1996.

[28] Guo Lin and Wan-Tong Li. Asymptotic spreading of competition diffusion systems: the role of interspecific competitions. European J. Appl. Math., 23(6):669-689, 2012.

[29] Aled Morris, Luca Börger, and Elaine C. M. Crooks. Individual variability in dispersal and invasion speed. ArXiv e-prints, dec 2016.

[30] Hans F. Weinberger Murray H. Protter. Maximum Principles in Differential Equations. Springer-Verlag, 1984.

[31] Emmanuel Risler. Competition between stable equilibria in reaction-diffusion systems: the influence of mobility on dominance. ArXiv e-prints, mar 2017.

[32] Min Ming Tang and Paul C. Fife. Propagating fronts for competing species equations with diffusion. Arch. Rational Mech. Anal., 73(1):69-77, 1980.

[33] Aizik I. Volpert, Vitaly A. Volpert, and Vladimir A. Volpert. Traveling wave solutions of parabolic systems, volume 140 of Translations of Mathematical Monographs. American Mathematical Society, Providence, RI, 1994. Translated from the Russian manuscript by James F. Heyda.

[34] Hans F. Weinberger, Mark A. Lewis, and Bingtuan Li. Analysis of linear determinacy for spread in cooperative models. J. Math. Biol., 45(3):183-218, 2002.

E-mail address: girardin@ljll.math.upmc.fr 